ARTICLE

\title{
Apoptotic stress-induced FGF signalling promotes non-cell autonomous resistance to cell death
}

Florian J. Bock (10) 1,2,3凶 , Egor Sedov , Elle Koren ${ }^{4}$, Anna L. Koessinger ${ }^{1,2}$, Catherine Cloix ${ }^{1,2}$, Désirée Zerbst ${ }^{1,2}$, Dimitris Athineos (10 1, Jayanthi Anand ${ }^{1}$, Kirsteen J. Campbell (1) ${ }^{1,2}$, Karen Blyth (1) ${ }^{1,2}$, Yaron Fuchs ${ }^{4} \&$ Stephen W. G. Tait (iD ${ }^{1,2}{ }^{凶}$

Damaged or superfluous cells are typically eliminated by apoptosis. Although apoptosis is a cell-autonomous process, apoptotic cells communicate with their environment in different ways. Here we describe a mechanism whereby cells under apoptotic stress can promote survival of neighbouring cells. We find that upon apoptotic stress, cells release the growth factor FGF2, leading to MEK-ERK-dependent transcriptional upregulation of pro-survival BCL2 proteins in a non-cell autonomous manner. This transient upregulation of pro-survival BCL2 proteins protects neighbouring cells from apoptosis. Accordingly, we find in certain cancer types a correlation between FGF-signalling, BCL-2 expression and worse prognosis. In vivo, upregulation of $\mathrm{MCL}-1$ occurs in an FGF-dependent manner during skin repair, which regulates healing dynamics. Importantly, either co-treatment with FGF-receptor inhibitors or removal of apoptotic stress restores apoptotic sensitivity to cytotoxic therapy and delays wound healing. These data reveal a pathway by which cells under apoptotic stress can increase resistance to cell death in surrounding cells. Beyond mediating cytotoxic drug resistance, this process also provides a potential link between tissue damage and repair.

\footnotetext{
${ }^{1}$ Cancer Research UK Beatson Institute, Garscube Estate, Switchback Road, Glasgow G61 1BD, UK. ${ }^{2}$ Institute of Cancer Sciences, University of Glasgow, Garscube Estate, Switchback Road, Glasgow G61 1QH, UK. ${ }^{3}$ Department of Radiotherapy (MAASTRO), GROW-School for Oncology and Developmental Biology, Maastricht University, 6229 ER Maastricht, The Netherlands. ${ }^{4}$ Laboratory of Stem Cell Biology and Regenerative Medicine, Department of Biology, Technion Israel Institute of Technology, Haifa 3200003, Israel.凶email: f.bock@maastrichtuniversity.nl; stephen.tait@glasgow.ac.uk
} 
T he cellular decision to live or die is fundamentally important in biology. Inappropriate cell survival has been causally linked to various diseases including cancer and autoimmunity ${ }^{1}$. In cancer, many therapies act by engaging apoptosis, and the degree of apoptotic sensitivity or apoptotic priming often correlates with therapeutic efficacy ${ }^{1,2}$. Therefore, understanding how cancer cells survive therapy should provide new ways to circumvent this and improve tumour cell elimination.

Mitochondrial apoptosis represents a major form of regulated cell death ${ }^{3}$. During apoptosis, mitochondria are permeabilised through a process called mitochondrial outer membrane permeabilisation or MOMP. Widespread MOMP effectively acts as cellular death sentence by releasing mitochondrial proteins, such as cytochrome $c$, that activate caspase proteases leading to rapid apoptosis $^{3}$. Even in the absence of caspases, MOMP typically commits a cell to death, and is thus considered a point-of-noreturn. Consequently, mitochondrial outer membrane integrity is tightly regulated by pro- and anti-apoptotic BCL-2 family proteins. Anti-apoptotic BCL-2 proteins prevent apoptosis by binding pro-apoptotic BAX, BAK and BH3-only proteins. During apoptosis, BH3-only proteins activate BAX and BAK, which subsequently promote MOMP. This process is exploited by $\mathrm{BH} 3-$ mimetics, a new class of anti-cancer drugs ${ }^{1}$. By binding antiapoptotic BCL-2 proteins, BH3-mimetics antagonise BCL-2 prosurvival function, sensitising cells to apoptosis ${ }^{4}$. Various BH3mimetics have been developed that target select or multiple antiapoptotic BCL-2 family members. Amongst them, the BCL-2 specific BH3-mimetic venetoclax ${ }^{5}$ shows considerable clinical promise and is approved for the treatment of chronic lymphocytic leukaemia (CLL) ${ }^{6}$ and in combination therapy to treat acute myeloid leukaemia (AML) ${ }^{7,8}$. However, in solid tumours, BH3mimetics are typically less effective, implying that additional survival mechanisms must be targeted in order to maximise their potential.

We set out to identify mechanisms of apoptotic resistance using BH3-mimetics as tool compounds. Selecting for cells surviving venetoclax treatment, we found that resistance was associated with increased anti-apoptotic BCL-2 and MCL-1 expression. Surprisingly, resistance occurred in a non-cell autonomous manner. We find that under apoptotic stress, cells can release FGF2. In turn, FGF2 triggers MEK-ERK signalling, resulting in increased anti-apoptotic BCL-2 and MCL-1 protein expression and apoptotic resistance. In certain cancer types, we found a correlation between FGF-signalling, BCL-2 and MCL-1 expression and poorer patient prognosis. Furthermore, we find FGF-dependent signalling results in upregulation of MCL-1 during wound healing and promotes tissue repair. Together, these findings unveil a non-cell autonomous mechanism of apoptotic resistance, where apoptotic stress-via FGF signalling-promotes cell survival. As we discuss, this process may have wide-ranging roles in health and disease.

\section{Results}

BH3-mimetics and BH3-only proteins upregulate BCL-2 and MCL-1 causing apoptotic resistance. We initially sought to define mechanisms of cell death resistance using BCL-2 targeting BH3-mimetics. For this purpose, we used our recently developed method called mito-priming 9 . In this system, cells co-express a pro-apoptotic BH3-only protein and an anti-apoptotic BCL-2 family member at equimolar levels and are therefore highly sensitive to BCL-2 targeting BH3-mimetic drugs (Fig. 1a). HeLa cells were used that stably express tBID together with BCL-2 (HeLa tBID-2A-BCL-2, hereafter called HeLa tBID2A). Cell viability was determined using livecell imaging following venetoclax treatment using Syto21 to label all cells and propidium iodide to label dead cells. As expected, the majority of cells died rapidly following venetoclax treatment, nevertheless some cells failed to die (Fig. 1b). To investigate the mechanisms of venetoclax resistance, HeLa tBID2A cells were cultured continuously in venetoclax to select for resistant cells. Increased expression of prosurvival BCL-2 family proteins is a common means of apoptotic resistance ${ }^{10}$. Indeed, cells that were continuously or intermittently cultured in venetoclax displayed higher expression of antiapoptotic BCL-2 and MCL-1 (Supplementary Fig. 1a). Surprisingly, following culture in regular medium post-venetoclax treatment, the resistant cells became sensitive to venetoclax again over time (Supplementary Fig. 1b). This decrease of resistance was accompanied by a decrease of BCL-2 and MCL-1 expression back to basal levels (Supplementary Fig. 1c). We next investigated whether short-term treatment with venetoclax was sufficient to promote BCL-2 and MCL-1 upregulation. Indeed, $3 \mathrm{~h}$ of venetoclax treatment led to increased levels of BCL-2 and MCL-1 (Fig. 1c). As before, BCL-2 and MCL-1 levels decreased following removal of venetoclax, demonstrating a reversible upregulation (Fig. 1d). This effect was not restricted to venetoclax, because an increase in BCL-2 and MCL-1 expression was also observed following treatment with other $\mathrm{BH} 3$ mimetics (navitoclax and ABT-737) (Supplementary Fig. 1d). Given that venetoclax-induced upregulation of BCL-2 and MCL-1 is reversible, this suggests that it is not genetically based. We noted an initial resistance of HeLa tBID2A cells cultured in venetoclax to re-treatment with venetoclax (Supplementary Fig. 1b), presumably due to increased levels of BCL-2 and MCL-1. Since increased levels of BCL-2 and MCL-1 were also observed in response to acute treatment, we investigated whether this was also sufficient to protect from apoptosis. Indeed, treatment with venetoclax for $48 \mathrm{~h}$ could protect from re-treatment with venetoclax and S63845, a specific inhibitor of MCL-11 (Fig. 1e). This protection was dependent on the increased levels of BCL-2 and MCL-1, because increasing the dose of venetoclax and S63845 could overcome the resistance (Fig. 1e). The pro-apoptotic proteins BAX and BAK are essential for mitochondrial outer membrane permeabilization (MOMP) during apoptosis ${ }^{12}$. To determine the role of apoptosis in the upregulation of BCL-2 and MCL-1, we generated HeLa tBID2A cells deficient in BAX and BAK using CRISPR-Cas9 genome editing. As expected, BAX BAK-deleted HeLa tBID2A cells were completely protected from mitochondrial apoptosis and caspase activation in response to venetoclax treatment (Supplementary Fig. 1e, f). Nevertheless, despite an inability to undergo apoptosis, BAX BAK-deleted HeLa tBID2A cells still upregulated BCL-2 and MCL-1 following venetoclax treatment (Fig. If, Supplementary Fig. 1g). Similarly, upregulation of BCL-2 and MCL-1 was also observed when caspase activity was blocked using the caspase inhibitor qVD-OPh (Fig. 1f). These data demonstrate that while the upregulation of BCL-2 and MCL-1 requires BH3-mimetic treatment, it occurs irrespective of apoptosis. Finally, to determine whether upregulation of BCL-2 and MCL-1 was specific to BH3-mimetics, we examined if a comparable effect could be observed by expressing BH3-only proteins. Control or BAX and BAK deleted HeLa cells were transfected with BH3-only proteins (tBID, PUMA, tBID (BIM BH3, with the BID BH3 domain replaced with the BIM BH3 domain)) and analysed for MCL-1 expression by western blot (Fig. 1g, Supplementary Fig. 1h). In all cases, MCL-1 expression was upregulated, indicating that $\mathrm{BH} 3$-only proteins can have similar effects as BH3-mimetics. Collectively, these data demonstrate that $\mathrm{BH} 3$-mimetics and $\mathrm{BH} 3$-only proteins can promote apoptotic resistance by increasing pro-survival BCL-2 protein expression. 
a

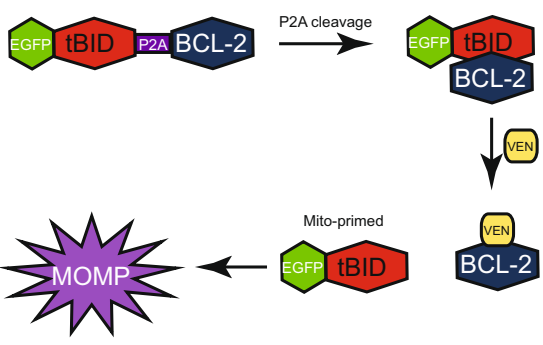

C

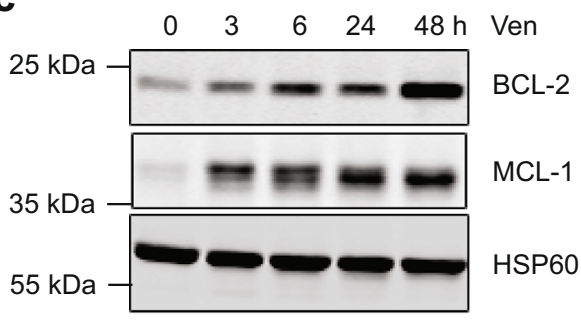

b

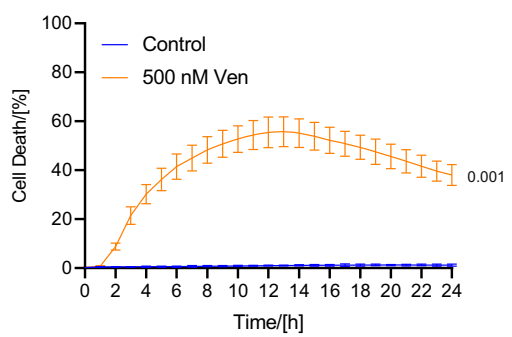

d

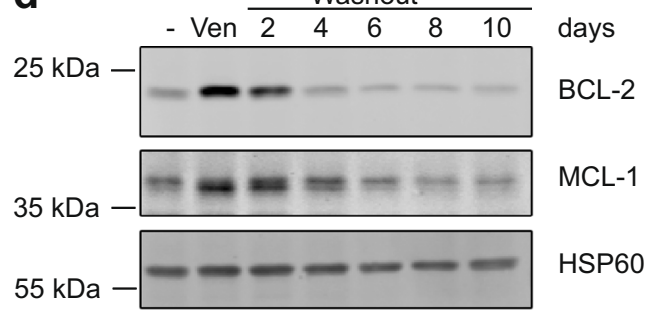

e

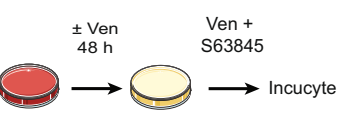

500 nM Ven + S63845

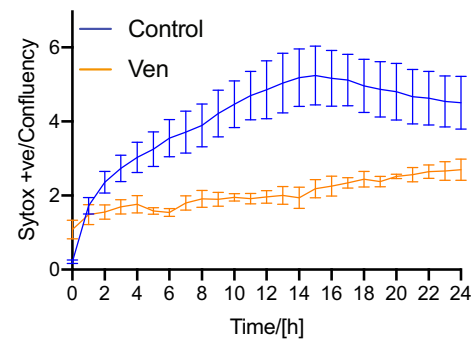

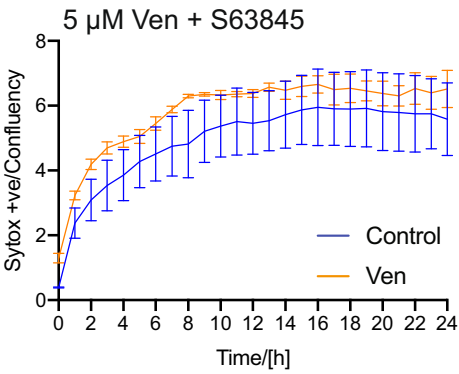

\section{f}

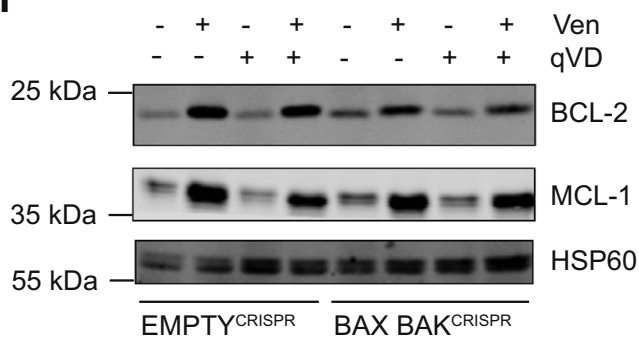

g
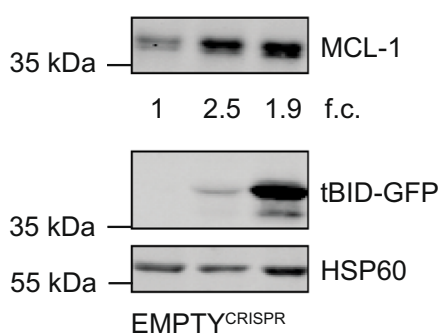

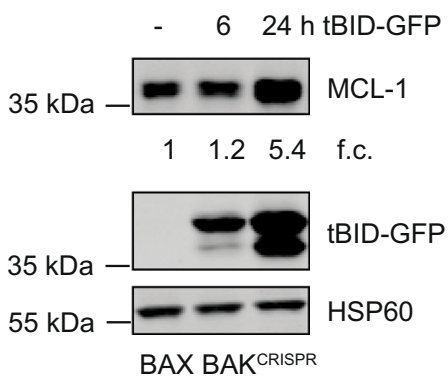

Fig. 1 BH3-mimetics and BH3-only proteins upregulate BCL-2 and MCL-1 causing apoptotic resistance. a Schematic model of the mitoprimed system. b HeLa tBID2A cells were treated with venetoclax (ven) and imaged over time. Percentage of dead cells was determined by staining all cells with Syto 21 and dead cells with propidium iodide. $n=3$ independent experiments; mean values \pm s.e.m.; unpaired, two-sided $t$-test at $24 \mathrm{~h}$. $\mathbf{c}$ HeLa tBID2A cells were treated with $500 \mathrm{nM}$ venetoclax, harvested at the indicated time points and protein expression was analysed by western blot (representative blot of three independent repeats). $\mathbf{d}$ HeLa tBID2A cells were treated for $48 \mathrm{~h}$ with $500 \mathrm{nM}$ venetoclax followed by replacement with regular growth medium (washout). At the indicated times post medium change cells were harvested and protein expression was analysed by western blot (representative blot of three independent repeats). e HeLa tBID2A cells were treated with or without venetoclax as indicated for $48 \mathrm{~h}$ followed by treatment with venetoclax and the MCL-1 inhibitor S63845. Cell death was then monitored by Sytox Green staining and Incucyte imaging. $n=3$ independent experiments; mean values \pm s.e.m. $\mathbf{f}$ Control or BAX BAKCRISPR HeLa tBID2A cells were treated with $500 \mathrm{nM}$ venetoclax in combination with $10 \mu \mathrm{M}$ qVD-OPh as indicated for $48 \mathrm{~h}$, harvested and protein expression was analysed by western blot (representative blot of three independent repeats). $\mathbf{g}$ Control or BAX BAKCRISPR HeLa cells were transfected with tBID-GFP plasmid, harvested after the indicated times and protein expression was analysed by western blot. Fold change normalised to loading control is stated below (representative blot of three independent repeats). 
a

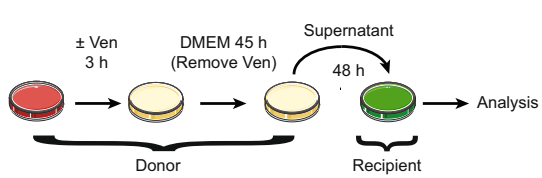

C

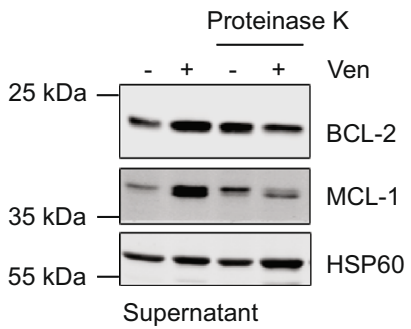

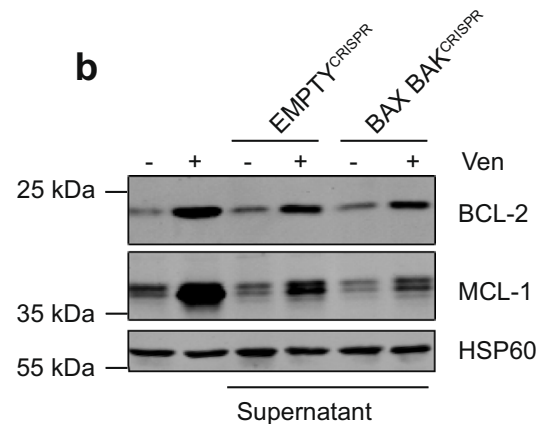

d

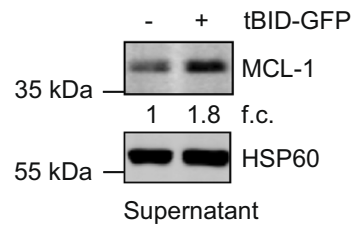

Fig. 2 BH3-mimetics and BH3-only proteins can upregulate anti-apoptotic BCL-2 proteins in a non-cell autonomous manner. a Schematic of supernatant transfer experiments: HeLa tBID2A cells were treated with $500 \mathrm{nM}$ venetoclax for $3 \mathrm{~h}$, followed by exchange to regular growth medium for $45 \mathrm{~h}$. Supernatant was harvested, filtered and added onto recipient cells before analysis. $\mathbf{b}$ HeLa tBID2A cells were treated directly with venetoclax or with supernatant from untreated or venetoclax treated control or BAX BAKCRISPR cells as described in $\mathbf{a}$. After $48 \mathrm{~h}$ cells were harvested and protein expression was analysed by western blot (representative blot of three independent repeats). c Supernatant from control or venetoclax treated cells was digested with Proteinase $\mathrm{K}$ before addition onto recipient cells and protein expression was analysed by western blot after $48 \mathrm{~h}$ (representative blot of three independent repeats). d Supernatant from HeLa cells transfected with tBID-GFP was collected after $48 \mathrm{~h}$ and transferred onto recipient HeLa cells. After $48 \mathrm{~h}$, recipient cells were harvested and protein expression analysed by western blot. Fold change normalised to loading control is stated below (representative blot of two independent repeats).

BH3-mimetics and $\mathrm{BH} 3-o n l y$ proteins can upregulate antiapoptotic BCL-2 proteins in a non-cell autonomous manner. To understand how venetoclax treatment causes upregulation of BCL-2 and MCL-1, we determined whether increases in protein or mRNA stability might contribute. HeLa tBID2A cells were treated with venetoclax for $24 \mathrm{~h}$, after which inhibitors of protein synthesis (cycloheximide) or transcription (actinomycin D) were added for varying times. Neither MCL-1 nor BCL-2 protein or mRNA stability was increased after venetoclax treatment (Supplementary Fig. 2a, b), indicating that mechanisms besides protein or mRNA stability are likely responsible. Given these results, we investigated if venetoclax might upregulate BCL-2 and MCL-1 in a non-cell autonomous manner. Control or BAX BAK deleted HeLa tBID2A cells were treated for $3 \mathrm{~h}$ with $500 \mathrm{nM}$ venetoclax, followed by exchange to regular medium for $45 \mathrm{~h}$. Media from treated cells was then transferred to recipient cells, which were examined for BCL-2 and MCL-1 expression after $48 \mathrm{~h}$ (Fig. 2a). Importantly, media from venetoclax treated HeLa tBID2A cells promoted upregulation of BCL-2 and MCL-1 in recipient cells (Fig. 2b). Similarly, supernatant from BAX and BAK deficient cells also promoted MCL1 and BCL-2 upregulation, supporting earlier findings that cell death is not required for this effect (Fig. 2b). Supernatant from venetoclax treated cells failed to induce apoptosis in recipient cells, demonstrating the absence of potentially residual venetoclax (Supplementary Fig. 2c). Additionally, media from HeLa tBID2A cells treated with a different BCL-2 inhibitor, S55746 ${ }^{13}$, also upregulated MCL-1 and BCL-2 in recipient cells (Supplementary Fig. 2d). To investigate the mechanism of this non-cell autonomous effect, we first characterised the signal causing upregulation of anti-apoptotic BCL-2 proteins. Supernatant from venetoclax treated HeLa tBID2A cells was subjected to centrifugal filtration using a filter with a $3 \mathrm{kDa}$ cut-off. Flow-through and concentrate were added to recipient cells for $48 \mathrm{~h}$, and MCL-1 and BCL-2 expression was determined by western blot (Supplementary Fig. 2e). Only the concentrate (containing molecules above $3 \mathrm{kDa}$ ) was capable of increasing BCL-2 and MCL-1 expression, suggesting that small molecules such as metabolites and lipids are not responsible. Importantly, Proteinase $\mathrm{K}$ treatment of supernatant from BH3-mimetic treated cells abolished the ability to upregulate MCL-1 and BCL-2, consistent with the factor(s) being proteinaceous (Fig. 2c). Finally, we investigated whether $\mathrm{BH} 3$-only proteins can also have a similar non-cell autonomous effect. HeLa or 293T cells were transfected with tBID and the supernatant was transferred onto recipient cells. Consistent with earlier results, supernatant transferred from tBID transfected cells also caused an up-regulation of BCL-2 and MCL-1 expression (Fig. 2d, Supplementary Fig. 2f). Together, these data demonstrate that $\mathrm{BH} 3$-mimetics and $\mathrm{BH} 3-$ only proteins can upregulate BCL-2 and MCL-1 expression in a non-cell autonomous manner.

Non-cell autonomous upregulation of anti-apoptotic BCL-2 proteins requires MEK-ERK signalling. We sought to define the non-cell autonomous mechanism causing anti-apoptotic BCL-2 protein upregulation. For this purpose, HeLa tBID2A cells were treated with venetoclax together with inhibitors targeting pathways previously implicated in anti-apoptotic BCL-2 regulation ${ }^{14-17}$. After co-treatment for $48 \mathrm{~h}$, cell lysates were probed for BCL-2 and MCL1 expression by western blot. Of all the tested inhibitors, only trametinib (a MEK kinase inhibitor ${ }^{18}$ ) potently blocked venetoclax induced BCL-2 and MCL-1 expression (Fig 3a, b). The decrease in phosphorylation of ERK1/2, a direct target of $\mathrm{MEK}^{19}$, validated trametinib activity (Fig. 3b). Upregulation of BCL-2 and MCL-1 was transcriptional, because venetoclax treatment increased RNA 


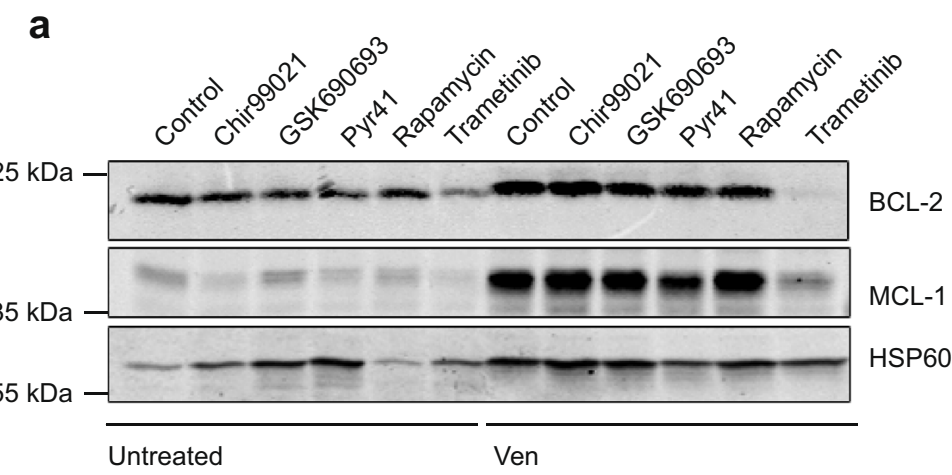

b

C

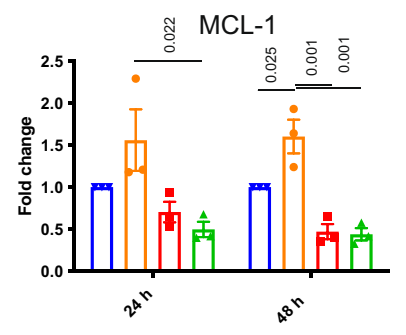

e

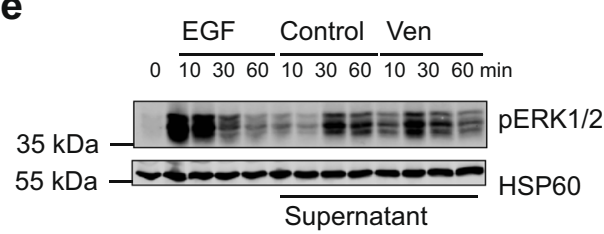

g

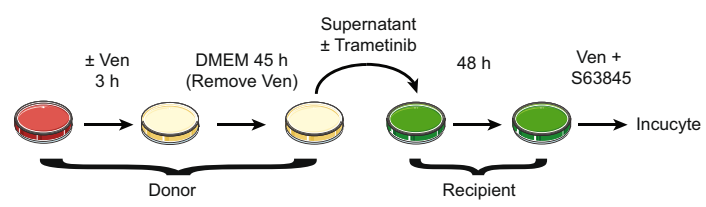

$500 \mathrm{nM}$ Ven + S63845

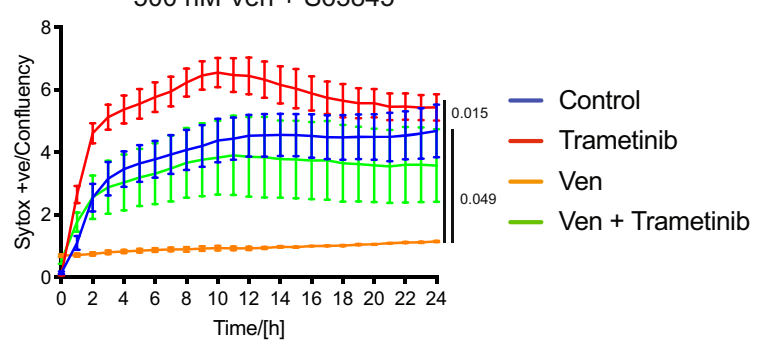

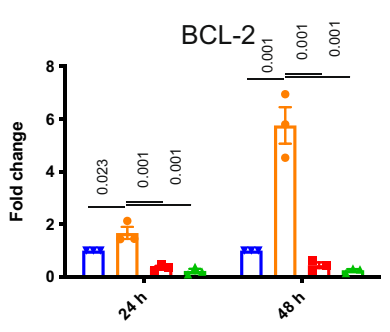

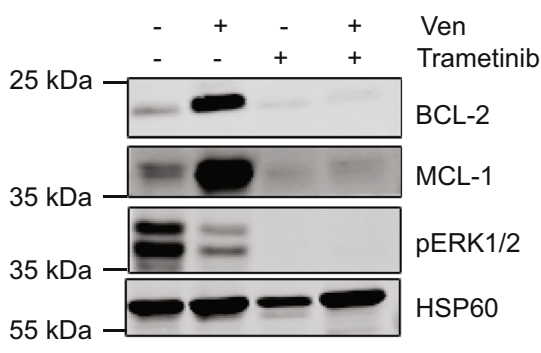

d

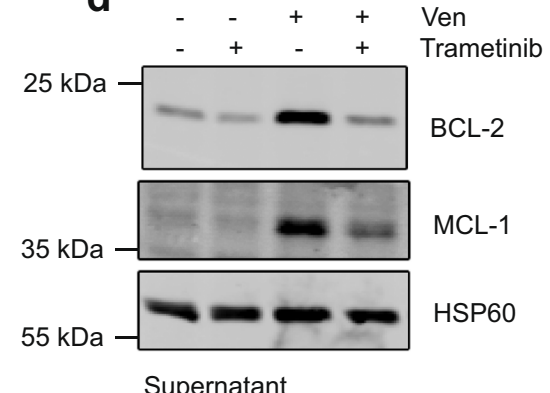

Supernatant
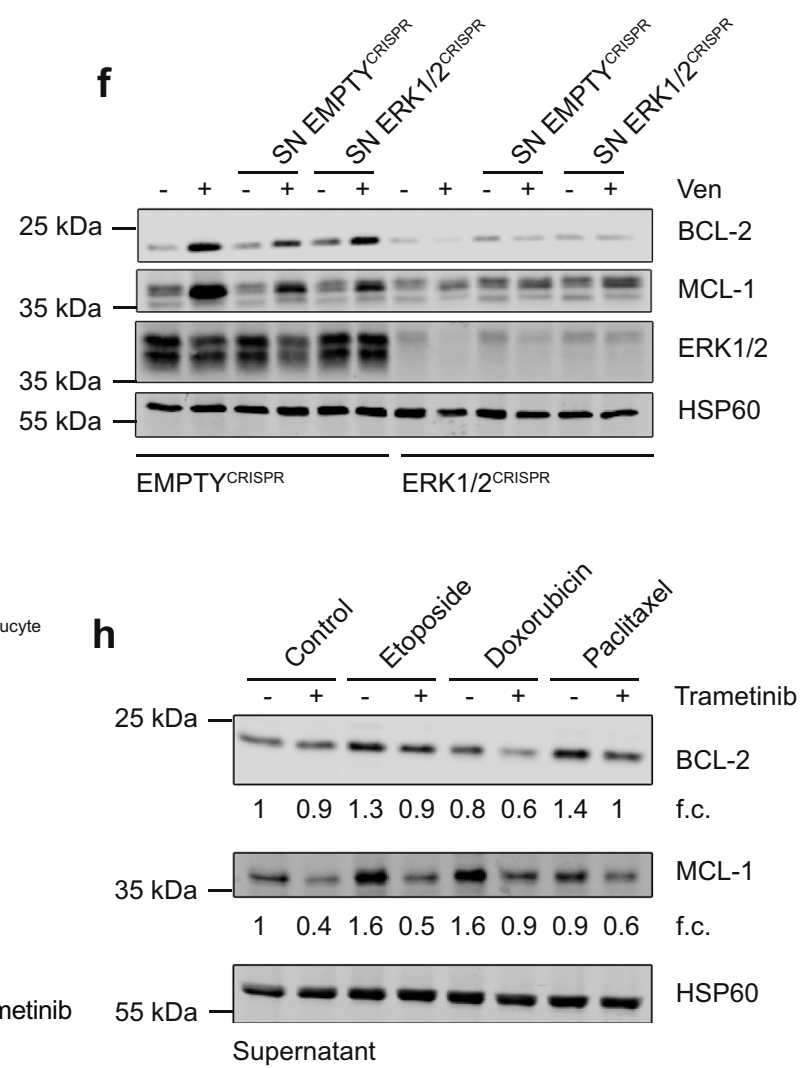

levels, which could be inhibited by trametinib co-treatment (Fig. 3c). The upregulation of MCL-1 by BH3-mimetics was not limited to HeLa cells, since it was also observed in IMR90 lung fibroblasts and CWR-R1 prostate cancer cells (Supplementary Fig. 3a, b). We next investigated whether non-cell autonomous upregulation of BCL-2 and MCL-1 required MEK-ERK signalling. Trametinib was added to supernatant from venetoclax treated HeLa tBID2A or CWR-R1 cells before the supernatant was added to recipient cells. BCL-2 and MCL-1 upregulation was effectively blocked by trametinib (Fig. 3d, Supplementary Fig. 3c). Again this 
Fig. 3 Non-cell autonomous upregulation of anti-apoptotic BCL-2 proteins requires MEK-ERK signalling. a HeLa tBID2A cells were untreated or treated with $500 \mathrm{nM}$ venetoclax in combination with the indicated inhibitors for $48 \mathrm{~h}$, harvested and protein expression was analysed by western blot (representative blot of two independent repeats). b HeLa tBID2A cells were untreated or treated with $500 \mathrm{nM}$ venetoclax in combination with $500 \mathrm{nM}$ trametinib for $48 \mathrm{~h}$, harvested and protein expression was analysed by western blot (representative blot of three independent repeats). c HeLa tBID2A cells were untreated or treated with $500 \mathrm{nM}$ venetoclax in combination with $500 \mathrm{nM}$ trametinib, harvested and RNA expression was analysed by RT-qPCR. $n=3$ independent experiments; mean values \pm s.e.m.; Tukey corrected one-way ANOVA. $\mathbf{d}$ Supernatant from untreated or venetoclax treated HeLa tBID2A cells was supplemented with $500 \mathrm{nM}$ trametinib before addition onto recipient cells. After $48 \mathrm{~h}$ of incubation, recipient cells were harvested and protein expression analysed by western blot (representative blot of three independent repeats). e Supernatant from untreated or venetoclax treated HeLa tBID2A cells was added onto recipient cells. After the indicated times, recipient cells were harvested and protein expression analysed by western blot. Treatment

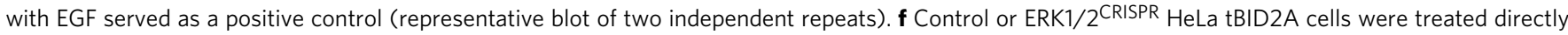

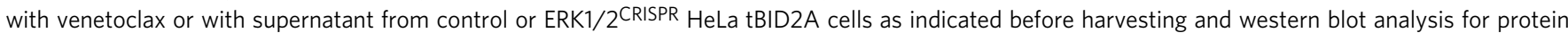
expression (representative blot of three independent repeats). $\mathbf{g}$ Supernatant from control or venetoclax treated HeLa tBID2A cells was supplemented with $500 \mathrm{nM}$ trametinib as indicated before addition onto recipient cells. After $48 \mathrm{~h}$ of incubation, the recipient cells were treated with $500 \mathrm{nM}$ venetoclax and 500 nM S63845 and survival was monitored by Sytox green exclusion and livecell imaging. $n=3$ independent experiments; mean values \pm s.e.m.; Tukey corrected one-way ANOVA. $\mathbf{h}$ HeLa cells were treated with the indicated drugs (Etoposide $50 \mu \mathrm{M}$, Doxorubicin $2 \mu \mathrm{M}$, Paclitaxel $1 \mu \mathrm{M}$ ) for $3 \mathrm{~h}$, followed by $45 \mathrm{~h}$ incubation in regular medium. Then the supernatant was harvested, filtered and supplemented or not with trametinib (500 $\mathrm{nM}$ ) before addition onto recipient cells. After $48 \mathrm{~h}$, cells were harvested and protein expression analysed by western blot. Fold change normalised to loading control is stated below (representative blot of three independent repeats).

effect was transcriptional, because upregulation of MCL-1 RNA in recipient cells was inhibited by trametinib addition to the supernatant (Supplementary Fig. 3d). Furthermore, supernatant of venetoclax treated cells could directly stimulate MEK activity in recipient cells as determined by increased pERK1/2 levels (Fig. 3e). To further investigate these findings, we generated ERK1/2 deficient HeLa tBID2A cells by CRISPR-Cas9 genome editing, hereafter called ERK1/2CRISPR cells (Fig. 3f). Media from control or ERK1/ $2^{\text {CRISPR }}$ deleted cells following venetoclax treatment was transferred onto control or ERK1/2CRISPR cells, and after $48 \mathrm{~h}$ BCL-2 and MCL-1 expression was determined by western blot. BCL-2 and MCL-1 expression increased following incubation with media from venetoclax treated cells or after direct treatment with venetoclax in control cells but was severely attenuated in ERK1/2CRISPR cells (Fig. 3f). Finally, we investigated whether MEK signalling, by enabling BCL-2 and MCL-1 upregulation, contributed to venetoclax resistance. HeLa tBID2A cells were incubated with venetoclax \pm trametinib for $48 \mathrm{~h}$, after which they were treated with venetoclax and S63845 and cell viability was determined by Sytox Green exclusion and Incucyte live-cell imaging (Supplementary Fig. 3e). Whereas venetoclax pre-treated cells were resistant, trametinib cotreatment completely abolished this resistance, supporting a functional role for MEK signalling in mediating apoptotic resistance via BCL-2 and MCL-1 upregulation. Similarly, transfer of venetoclax treated supernatant conferred resistance to the recipient cells. The resistance was dependent on BCL-2 and MCL-1 upregulation because supplementing the venetoclax treated supernatant with trametinib before addition to recipient cells re-sensitised those cells to the cytotoxic treatment (Fig. 3g). We next investigated if non-cell autonomous upregulation of BCL-2 and MCL-1 was specific to $\mathrm{BH} 3$-mimetics or could also be triggered by conventional chemotherapies. Similar to previous experiments, we treated HeLa cells with different chemotherapeutic drugs (etoposide, doxorubicin and paclitaxel) before transferring the supernatant to recipient cells. All three drugs promoted BCL-2 and MCL-1 upregulation, suggesting that apoptosis-inducing stresses can generally induce this effect (Fig. 3h). Supplementing the supernatant with trametinib before addition to recipient cells prevented upregulation, demonstrating that MEK-ERK signalling is essential for the upregulation, consistent with our earlier data. Collectively, these data show that apoptosis-inducing stresses activate MEK-ERK signalling, causing upregulation of BCL-2 and MCL1 and apoptotic resistance in a non-cell autonomous manner.

FGF signalling mediates non-cell autonomous upregulation of BCL-2 proteins. Various ligands can bind to receptors that signal through MEK-ERK, with receptor tyrosine kinases (RTK) being prominent activators of this pathway. Therefore, to determine the paracrine mediator(s) causing BCL-2 and MCL-1 upregulation, we initially focussed on RTK pathways. To identify potential ligands present after $\mathrm{BH} 3$-mimetic treatment, supernatant from control or venetoclax treated HeLa tBID2A cells was incubated with a human growth factor antibody array enabling detection of 41 different growth factors (Fig. 4a). Of the growth factors present on the array, FGF2 was increased following venetoclax treatment (Fig. 4a). Upregulation of FGF2 in the supernatant was confirmed by subsequent ELISA analysis (Fig. 4b). Addition of recombinant FGF2 to cells at a concentration similar to what we measured in venetoclax treated supernatant was sufficient to upregulate BCL-2 and MCL-1 expression (Fig. 4c). To directly test the importance of FGF2 in mediating paracrine upregulation of BCL-2 proteins, we generated FGF2 deficient cell lines by CRISPR-Cas9 (Supplementary Fig. 4a). Loss of FGF2 completely suppressed the ability of venetoclax to upregulate BCL-2 and MCL-1 in a paracrine manner, supporting a key role for FGF2 (Fig. 4d). Consistent with activation of FGF-signalling, known target genes of FGF receptors $\left(\mathrm{CDX}^{20}, \mathrm{DUSP}^{21}\right.$ and SPRY $\left.4^{22}\right)$ were also upregulated in response to supernatant from venetoclax treated cells (Fig. 4e). This upregulation could be blocked by adding trametinib to the supernatant before addition to recipient cells. To investigate the requirement of FGF receptors for upregulation of BCL-2 and MCL-1, we used two different FGFR inhibitors (AZD4547 and PRN137123,24). Co-treatment of HeLa tBID2A cells with either inhibitor and venetoclax prevented BCL-2 and MCL-1 upregulation (Supplementary Fig. 4b). Similarly, supernatant from venetoclax treated HeLa tBID2A cells supplemented with FGFR inhibitors prevented upregulation of BCL-2 and MCL-1 on recipient cells (Fig. 4f). Reduced upregulation of MCL1 was also observed upon co-treatment of MRC-5 lung fibroblast cells with venetoclax and PRN1371 (Supplementary Fig. 4c). The FGF receptor family is composed of four different receptors ${ }^{25}$, of which RNAseq analysis showed that FGFR2 was barely expressed in the HeLa cells used here (Supplementary Fig. 4d). To determine which receptors were responsible to signal the upregulation of BCL-2 and MCL-1 by FGF2, we used RNAi to knock down their expression individually or in combination. Knocking down FGFR1 and FGFR3, either individually or in combination, prevented upregulation of BCL-2 and MCL-1 either after direct treatment (Fig. 4g, Supplementary Fig. 4e) or with venetoclax treated supernatant (Fig. 4h). In contrast, knockdown of FGFR4 failed to affect BCL-2 and MCL-1 expression (Supplementary Fig. $4 \mathrm{f}-\mathrm{h}$ ). We next aimed to understand how FGF2 is regulated 
a

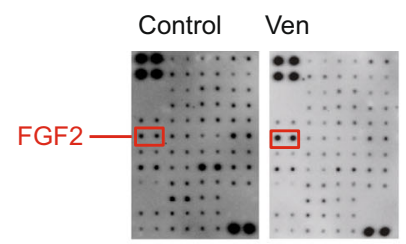

d

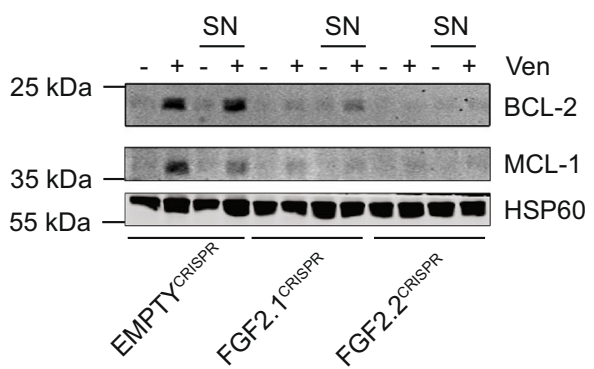

g

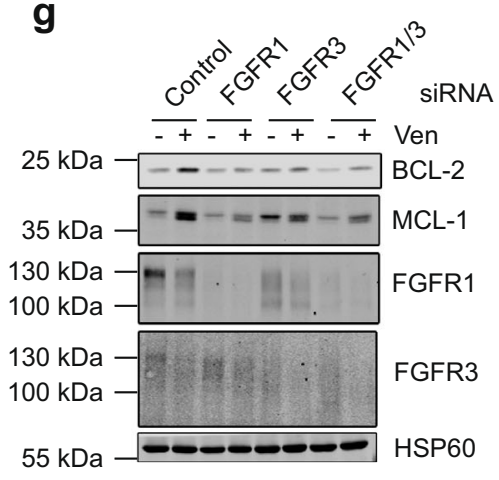

b

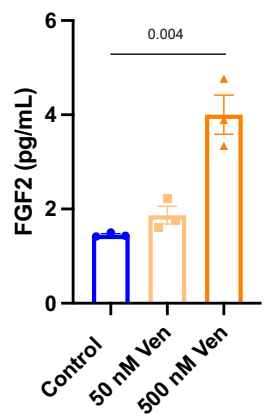

e

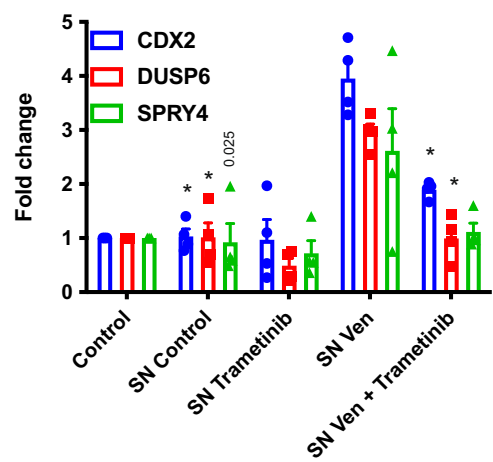

h

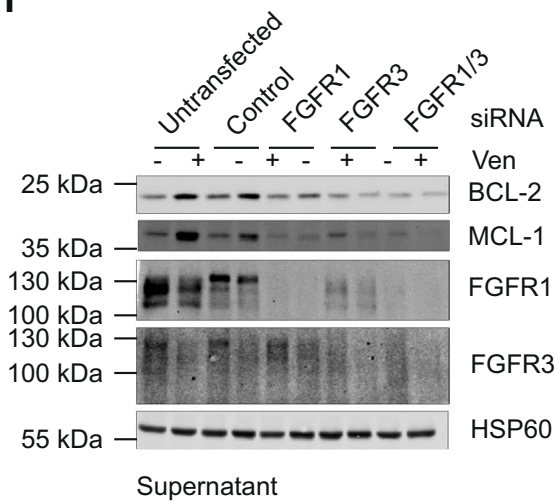

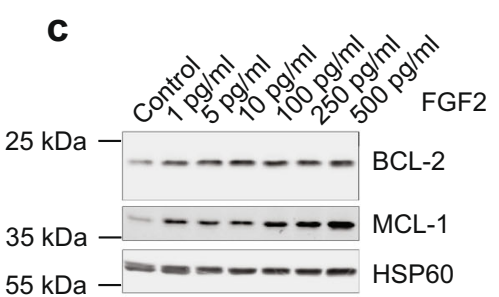

f

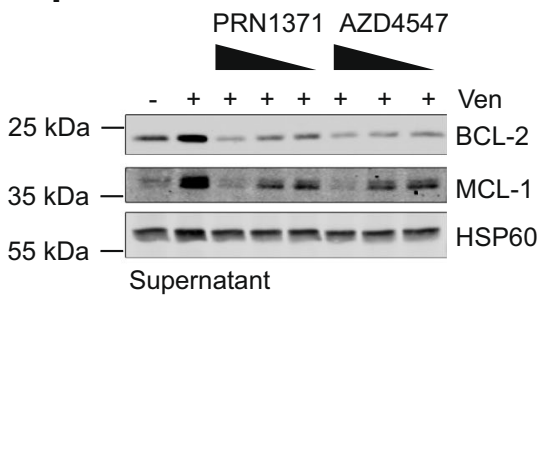

Fig. 4 FGF signalling mediates non-cell autonomous upregulation of BCL-2 proteins. a A growth factor membrane ligand array was probed with supernatant from control or venetoclax treated HeLa tBID2A cells. Spots for FGF2 are indicated. $\mathbf{b}$ Levels of FGF2 were determined in supernatant from control or venetoclax treated HeLa tBID2A cells by ELISA. $n=3$ independent experiments; mean values \pm s.e.m.; unpaired, two-sided $t$-test. $\mathbf{c}$ HeLa tBID2A cells were treated with recombinant FGF2, harvested after $6 \mathrm{~h}$ and protein expression analysed by western blot (representative blot of three independent repeats). d Control or FGF2CRISPR HeLa tBID2A cells were directly treated with venetoclax or the respective supernatant for $48 \mathrm{~h}$ as indicated before harvesting and analysis of protein expression by western blot (representative blot of three independent repeats). e Supernatant from untreated or venetoclax treated HeLa tBID2A cells was supplemented with $500 \mathrm{nM}$ trametinib before addition onto recipient cells. After $3 \mathrm{~h}$, recipient cells were harvested and RNA expression of FGF target genes analysed by RT-qPCR. $n=4$ independent experiments; mean values \pm s.e.m.; ${ }^{\star} p<0.0001$ compared to SN Ven; Dunnetts corrected one-way ANOVA. f Supernatant from control or venetoclax treated HeLa tBID2A cells was supplemented with decreasing doses of FGFR inhibitors as indicated (AZD: $5 \mu \mathrm{M}, 2.5 \mu \mathrm{M}, 1.25 \mu \mathrm{M}$; PRN1371: $10 \mu \mathrm{M}, 5 \mu \mathrm{M}, 2.5 \mu \mathrm{M}$ ) before addition onto recipient cells for $48 \mathrm{~h}$ and analysis of protein expression by western blot (representative blot of three independent repeats). $\mathbf{g}$ HeLa tBID2A cells were transfected with siRNA targeting FGFR1 and FGFR3 alone or in combination for $24 \mathrm{~h}$ before addition of $500 \mathrm{nM}$ venetoclax, harvesting after $48 \mathrm{~h}$ and analysis of protein expression by western blot (representative blot of three independent repeats). $\mathbf{h}$ HeLa tBID2A cells were transfected with siRNA targeting FGFR1 and FGFR3 alone or in combination for $24 \mathrm{~h}$ before addition of control or venetoclax treated supernatant from control cells, harvesting after $48 \mathrm{~h}$ and analysis of protein expression by western blot (representative blot of three independent repeats).

in response to $\mathrm{BH} 3$-mimetic treatment. A minor, yet significant increase in FGF2 mRNA was detected in both wild type and BAX BAK deficient HeLa tBID2A cells following venetoclax treatment (Fig. 5a), however there was a decrease in FGF2 protein levels over time (Fig. 5b). Given the lack of increase in FGF2 protein level, we investigated whether inhibiting transcription in donor cells affected the upregulation of BCL-2 and MCL-1 in recipient cells. Supernatant from cells co-treated with venetoclax and actinomycin D still led to upregulation of MCL-1 and BCL-2 after transfer (Fig. 5c), suggesting that transcriptional regulation is not necessary. In contrast, actinomycin D prevented upregulation of MCL1 in supernatant-treated recipient cells (Fig. 5d), corroborating transcriptional upregulation of $\mathrm{MCL}-1$ in recipient cells. These experiments demonstrate that the activation of FGF2 in response to apoptotic stress is independent of induced expression, but instead may be due to increased release from the cell. Collectively, these data demonstrate that FGF-signalling, triggered by release of FGF2 from BH3-mimetic treated cells, is required and 
a

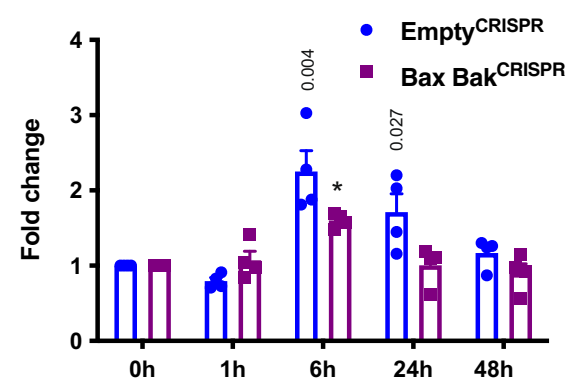

C

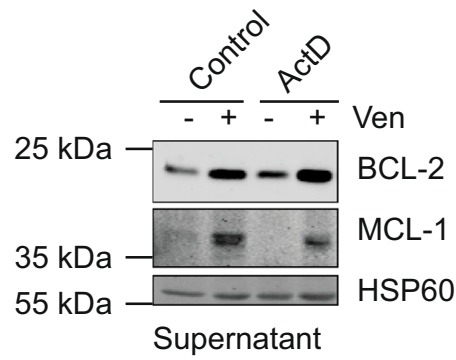

b

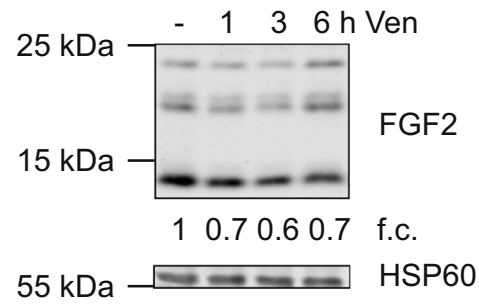

d

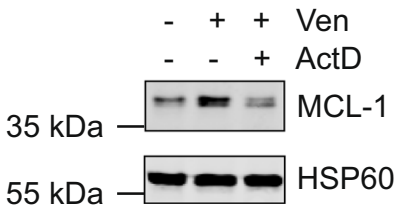

Fig. 5 Non-cell autonomous upregulation of BCL-2 proteins is independent of transcription. a HeLa tBID2A cells were treated with $500 \mathrm{nM}$ venetoclax for the indicated time, RNA was harvested and expression of FGF2 mRNA analysed by qPCR. $n=4$ independent experiments; mean values \pm s.e.m.; ${ }^{\star} p<0.0001$; unpaired, two-sided $t$-test. $\mathbf{b}$ HeLa tBID2A cells were treated with $500 \mathrm{nM}$ venetoclax for the indicated time, harvested and protein expression analysed by western blot. Fold change normalised to loading control is stated below (representative blot of three independent repeats). c HeLa tBID2A cells were treated with venetoclax \pm actinomycin $D$ for $3 \mathrm{~h}$, followed by $45 \mathrm{~h}$ incubation in regular medium. Then the supernatant was harvested, filtered and added onto recipient cells. After $48 \mathrm{~h}$, recipient cells were harvested and protein expression analysed by western blot (representative blot of three independent repeats). $\mathbf{d}$ HeLa tBID2A cells were treated with venetoclax for $3 \mathrm{~h}$, followed by $45 \mathrm{~h}$ incubation in regular medium. Then the supernatant was harvested, filtered, supplemented with actinomycin D as indicated and added onto recipient cells. After $3 \mathrm{~h}$, recipient cells were harvested and protein expression analysed by western blot (representative blot of three independent repeats).

sufficient for non-cell autonomous upregulation of anti-apoptotic BCL-2 proteins.

FGF signalling is essential for non-cell autonomous apoptotic resistance. We next investigated the contribution of FGFmediated BCL-2 and MCL-1 upregulation to apoptosis resistance. First, HeLa tBID2A cells were co-treated with venetoclax and inhibitors of FGF signalling (PRN1371, AZD4547) or MEK (trametinib). Whereas the venetoclax treated cells were resistant to apoptosis induced by re-treatment with venetoclax and S63845, cells co-treated with FGF signalling inhibitors were resensitised to apoptosis (Fig. 6a, Supplementary Fig. 5a). As observed previously, increasing the dose of venetoclax and S63845 could restore sensitivity to venetoclax pretreated cells. Next, the potential of FGF signalling to promote non-cell autonomous apoptotic resistance was investigated. Supernatant was harvested from venetoclax treated HeLa tBID2A cells and supplemented with inhibitors of the FGF signalling pathway before addition onto recipient cells. Consistent with our earlier data, supernatant from venetoclax treated cells conferred apoptotic resistance to recipient cells (Fig. 6b, Supplementary Fig. 5b). Crucially, apoptotic sensitivity was restored upon inhibition of either FGF or MEK signalling. Again, increasing the dose of venetoclax and S63845 could kill the venetoclax pretreated resistant cells. To investigate this further, we assessed effects on long-term clonogenic survival. Supporting earlier data, supernatant from venetoclax treated cells could confer long-term protection from apoptosis, which was restored by supplementation of FGF signalling inhibitors (Fig. 6c). To further investigate these findings, we transferred supernatant from venetoclax treated control or FGF2 deficient cells
(Supplementary Fig. 4a) and tested survival in response to venetoclax and S63845. While the supernatant from the wildtype cells protected from apoptosis, this protection was attenuated in cells treated with supernatant from FGF2 deficient cells (Fig. 6d). Increasing the concentration of venetoclax and S63845 could overcome the protective effect of supernatant from venetoclax treated cells. These data support a model whereby following apoptotic stress, cells can signal non-cell autonomous apoptotic resistance by FGF-signalling dependent on the upregulation of BCL-2 and MCL-1 (Fig. 6e). Finally, we investigated whether a similar mechanism may be evident in cancer. Interrogating a total of 25 different TCGA cancer datasets, we first removed patients with mutations in components of the FGFR signalling pathway. These patients might show an altered activation of the FGF signalling pathway independent of the non-cell autonomous pathway described in this work. We next calculated an FGF pathway activation score by determining the mean expression of ten FGF target genes as a proxy for activation of FGF signalling. The FGF score was then correlated with MCL-1 and BCL-2 expression to determine if increased activation of FGF signalling correlates with increased levels of prosurvival BCL-2 family gene expression. Using this approach, we identified several cancer types that displayed a correlation between FGF activation and BCL-2 and/ or MCL-1 expression (Fig. 6f, Supplementary Fig. 6a, b). Next, we determined whether this correlation had an influence on disease progression by stratifying patients into groups based on FGF score and BCL- 2 or MCL-1 expression. Strikingly, in three out of 25 tested cancer types, survival of the high scoring group (high activation of FGF signalling and high expression of BCL-2 or MCL-1) was significantly decreased when compared to the 
a
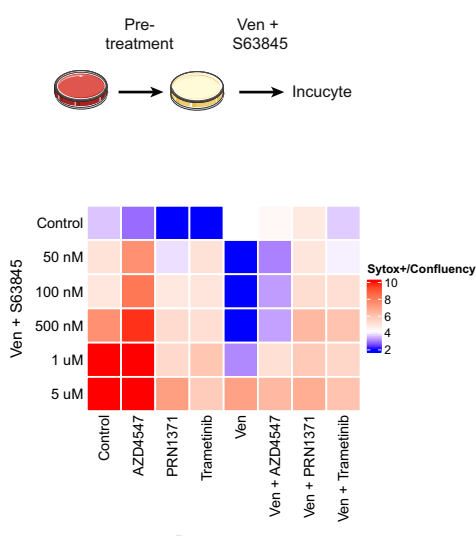

Pretreatment b
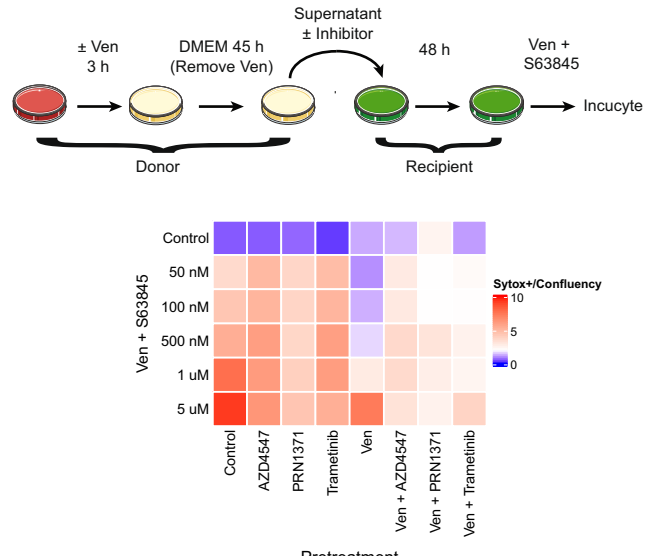

C

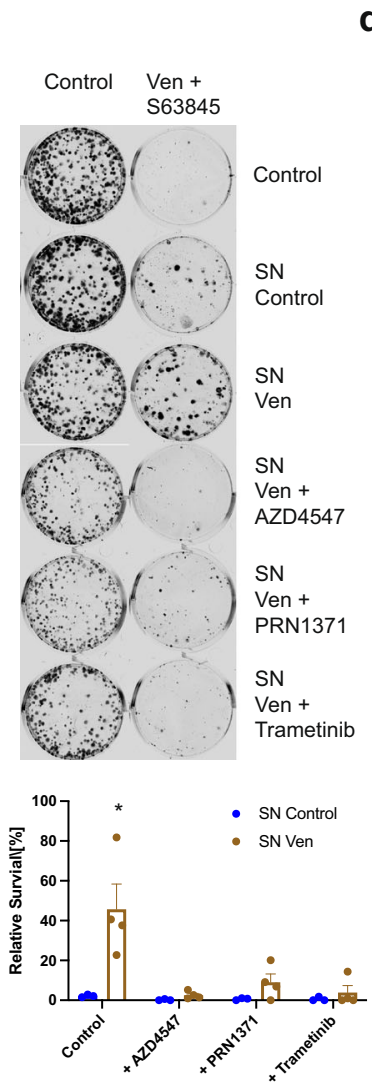

d

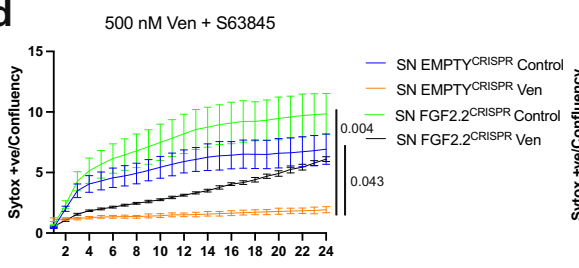

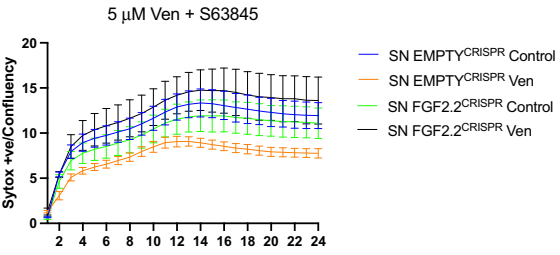

e

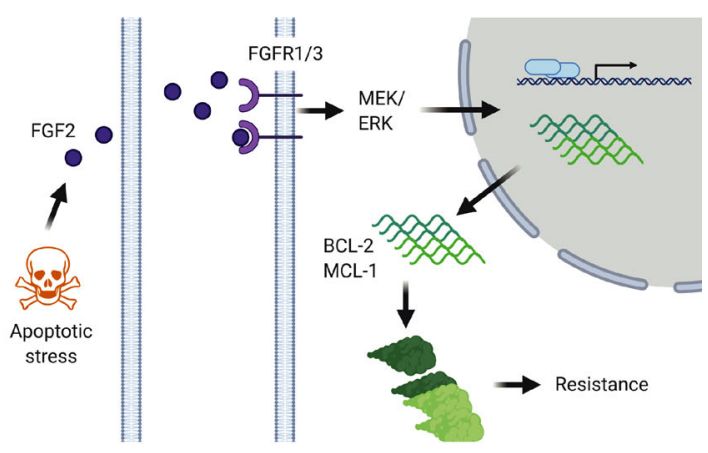

f Thymoma
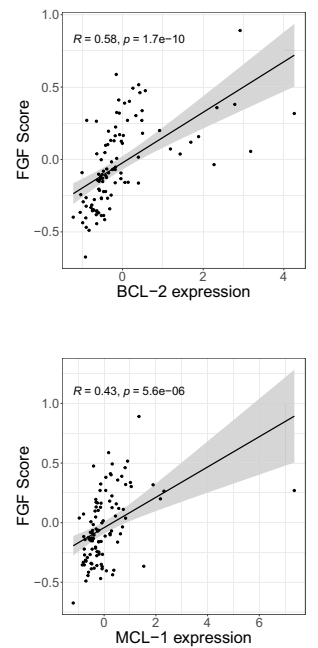
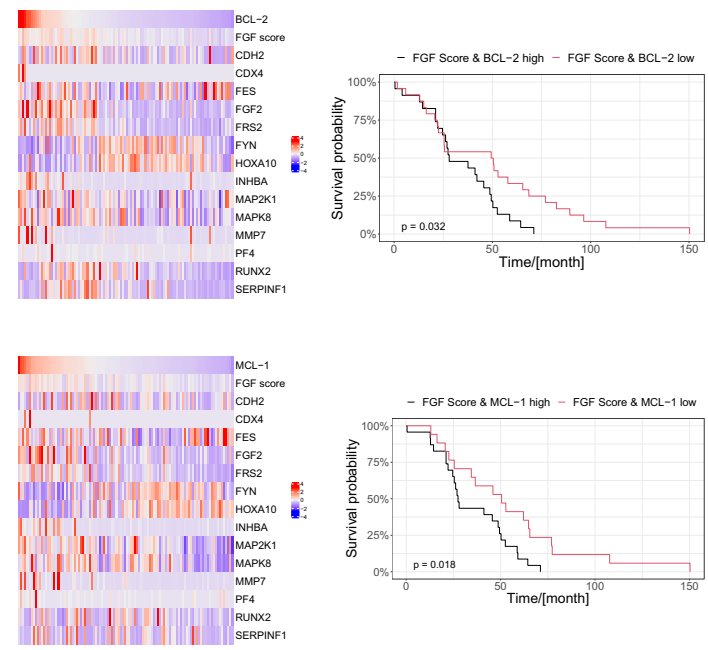

low scoring group (Fig. 6f, Supplementary Fig. 6a, b). These data suggest that FGF-mediated resistance might have a protective effect on cancer cell survival and therefore worsen prognosis.
FGF signalling induces MCL-1 in the skin and regulates wound healing dynamics. Oncogenic processes are often subverted homoeostatic mechanisms. We therefore sought to define physiological roles for FGF-signalling induced anti-apoptotic BCL-2 
Fig. 6 FGF signalling is essential for non-cell autonomous apoptotic resistance. a HeLa tBID2A cells were treated with $500 \mathrm{nM}$ venetoclax in combination with RTK pathway inhibitors (AZD4547 (5 $\mu$ M), PRN1371 $(10 \mu \mathrm{M})$ or trametinib $(500 \mathrm{nM})$ ) as indicated for $48 \mathrm{~h}$. Then the cells were treated with the indicated concentrations venetoclax $+\mathrm{S} 63845$ and cell survival was monitored by Incucyte. Heatmap colours show cell death at $24 \mathrm{~h}$ (mean from $n=3$ independent experiments), corresponding survival curves are shown in Supplementary Fig. 5a. b HeLa tBID2A cells were incubated with 500 nM venetoclax treated supernatant supplemented with RTK pathway inhibitors (AZD4547 (5 $\mu$ M), PRN1371 (10 $\mu$ M) or trametinib (500 nM)) as indicated before addition onto recipient cells for $48 \mathrm{~h}$. Then the cells were treated with the indicated concentrations venetoclax $+\mathrm{S} 63845$ and cell survival was monitored by Incucyte. Heatmap colours show cell death at $24 \mathrm{~h}$ (mean from $n=3$ independent experiments), corresponding survival curves are shown in Supplementary Fig. 5b. c HeLa tBID2A cells were plated at low density (1000 cells per 6 well) and incubated with 500 nM venetoclax-treated supernatant supplemented with RTK pathway inhibitors (AZD4547 $(5 \mu \mathrm{M})$, PRN1371 $(10 \mu \mathrm{M})$ or trametinib $(500 \mathrm{nM})$ ) as indicated for $48 \mathrm{~h}$. Cells were then treated with $2.5 \mu \mathrm{M}$ venetoclax $+\mathrm{S} 63845$ for another $48 \mathrm{~h}$ before replacement with regular growth medium. After an additional 5 days colonies were visualised by crystal violet staining and quantified. $n \geq 3$ independent experiments; mean values \pm s.e.m.; ${ }^{*} p<0.05$ compared to all other treatments; Tukey corrected one-way ANOVA. d Control or FGF2 deficient cells were treated for $3 \mathrm{~h}$ with $500 \mathrm{nM}$ venetoclax followed by $45 \mathrm{~h}$ regular medium. Then the supernatant was harvested and added to control cells for $48 \mathrm{~h}$. After that, the cells were treated with venetoclax $+\mathrm{S} 63845$ with the indicated doses and survival monitored by Incucyte. $n=3$ independent experiments; mean values \pm s.e.m.; ${ }^{*} p<0.05$ compared to venetoclax treatment at 24 h; Tukey corrected oneway ANOVA. e Working model. f Pearson correlation between FGF Score and BCL-2 (upper left) or MCL-1 (lower left) in the TCGA Thymoma dataset ( \pm s.e.m, 105 patients). FGF score, FGF receptor target gene and BCL-2 (upper middle) or MCL-1 (lower middle) expression in the TCGA Thymoma dataset. Survival of TCGA Thymoma patients stratified by FGF score and BCL-2 (upper right) or MCL-1 (lower right) expression ( $p$ value was calculated with a logrank test).

expression. Because our data are consistent with a model in which stressed cells can alert the microenvironment to increase their threshold against apoptosis, we decided to test this hypothesis in a physiologically relevant and experimentally tractable model. In the epidermis, apoptosis has been found to play an important role in skin repair ${ }^{26,27}$. Notably, re-epithelization of the skin relies heavily on FGF signalling ${ }^{28}$. We therefore set out to examine whether FGF signal transduction might also modulate MCL-1 expression in the skin upon wound infliction. We hypothesised that an injured tissue might increase its apoptotic threshold to limit damage due to cell death in areas that require regeneration. This increased resistance to apoptosis could potentially protect against excessive cell death which could hinder tissue regeneration. Indeed, subjecting the dorsal skin of mice to $1 \mathrm{~cm}^{2}$ fullthickness excisional wounds promoted upregulation of MCL-1 in keratinocytes in the vicinity of the wound (Fig. 7a). We next examined whether inhibition of FGF signalling affected MCL-1 expression by utilising the FGFR inhibitor AZD4547 and the MEK inhibitor trametinib. We administered the inhibitors for four consecutive days by subcutaneous injection prior to infliction of the wound and harvested the skin three days post wound infliction (PWI). Our results indicate that administration of either inhibitor dramatically decreased the number of MCL-1 + cells following injury (Fig. $7 \mathrm{~b}, \mathrm{c}$ ), suggesting that upregulation of MCL-1 in response to skin injury is dependent on FGF signalling.

To investigate the physiological role of FGF mediated MCL-1 upregulation, we monitored wound closure dynamics. For this aim, we inflicted full-excisional wounds on dorsal skins of control or inhibitor treated mice and evaluated wound coverage at specific time points PWI (Supplementary Fig. 7a). In control mice, the wound size was reduced by $\sim 40 \%$ after just one day, whereas in inhibitor treated mice no coverage was seen at this time point (Fig. 7d). This delay was accompanied by a decreased area of re-epithelization (Fig. 7d, Supplementary Fig. 7b, c). To examine the contribution of epidermal keratinocytes to the observed phenotypes, we next evaluated proliferation in the wound bed. We harvested wounds at three and seven days PWI. FGFR and MEK-inhibition resulted in decreased proliferation evident by $\mathrm{Mcm} 2$ and Ki67 immunostaining (Supplementary Fig. $7 b, d, e)$. The attenuation in wound closure phenotypes seen upon FGFR-inhibition could potentially be facilitated via decreased basal cell expansion and suprabasal migration capacity. Elegant studies have shown that in early stages of wounding healing the leading edge of the wound is mostly composed of non-proliferative migratory cells that can drive rapid re- epithelialization 29,30 . Our analysis revealed that FGFR-inhibited wounds displayed a less pronounced leading edge by seven days PWI, when compared to control (Supplementary Fig. 7b, c). These findings suggest that suprabasal keratinocytes may also contribute to the attenuated wound healing phenotypes seen upon inhibition of FGF receptors and MEK. Our data indicate that FGF signalling induces the expression of MCL-1 in the skin and affects the contribution of both basal and suprabasal keratinocytes to the wound repair process. Overall, our results suggest a mechanism in which cells protect their tissue integrity by increasing the apoptotic threshold in response to stress by FGF2 mediated upregulation of pro-survival proteins.

\section{Discussion}

Innate or acquired resistance to cell death is of fundamental importance in health and disease. For instance, evasion of apoptosis can both promote cancer and inhibit treatment response, leading to tumour relapse $\mathrm{s}^{31}$. To understand how cells can resist cell death, we used BCL-2 targeting BH3-mimetics as tool compounds. Unexpectedly, we uncovered a non-cell autonomous mechanism that enables apoptotic resistance. We found that upon apoptotic stress, cells can release the growth factor FGF2. By activating MEK-ERK signalling, FGF2 upregulates antiapoptotic BCL-2 protein expression ${ }^{32,33}$ in neighbouring cells, protecting against apoptosis. Importantly, resistance can be overcome by co-treatment of $\mathrm{BH} 3$-mimetics with FGFR-inhibitors, demonstrating the functional significance of the pathway. Accordingly, in an in vivo injury model inhibition of FGFR signalling prevents MCL-1 upregulation, apoptosis and successively wound healing. Finally, we describe a correlation between increased FGF signalling, anti-apoptotic BCL-2 protein expression and poor patient prognosis, suggesting its relevance in vivo. As we discuss further, the process we describe here, where cell death promotes life, may have various pathophysiological functions.

Most cancer therapies work by killing tumour cells, consequently resistance to cell death profoundly impacts on therapeutic efficacy ${ }^{31}$. Typically, cancer cells can evade apoptosis through inactivating mutations in pathways that sense damage or activate cell death ${ }^{31}$. For instance, in chronic lymphocytic leukaemia (CLL), BCL-2 mutations have recently been reported that render BCL-2 unable to effectively bind the $\mathrm{BH} 3$-mimetic venetoclax, causing apoptotic resistance ${ }^{34-37}$. The resistance mechanism we report here is not mutation based, but instead is due to dying cells releasing FGF2 that causes transient apoptotic resistance in 
a
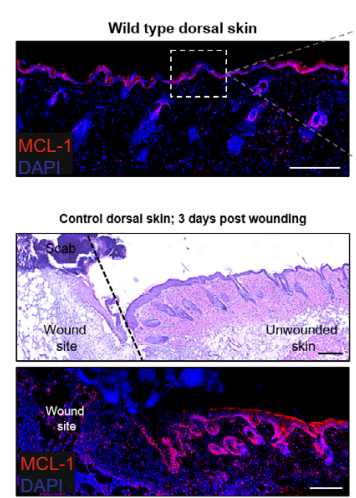

b

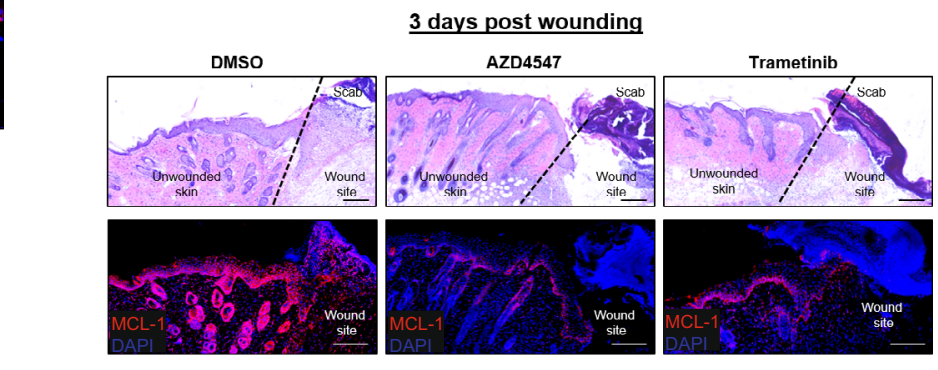

C

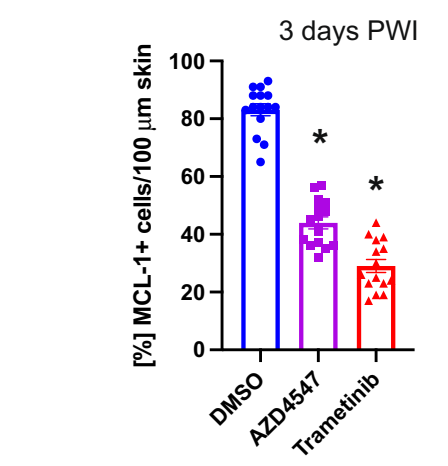

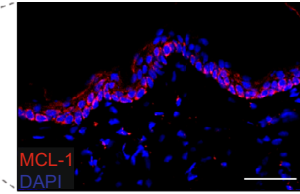

Control dorsal skin; 7 days post wounding

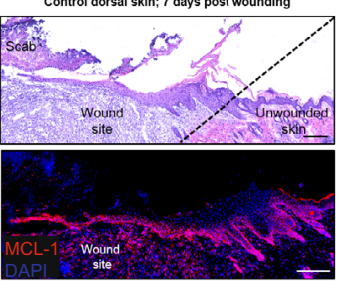

d

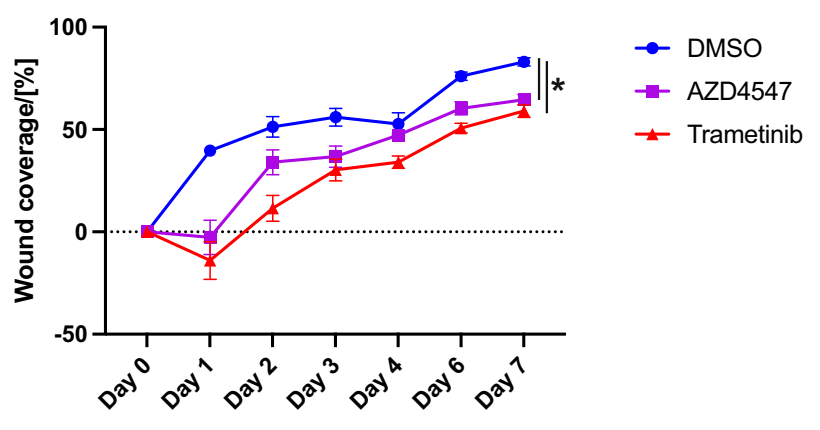

Fig. 7 FGFR and MEK inhibition decreases MCL-1 expression and hinders wound repair. a $\mathrm{H} \& \mathrm{E}$ and immunostaining of $\mathrm{MCL}-1$ in unwounded skins and 3 or 7 days post wounding (PWI). Representative image of $n=15$ mice, Scale bar: $200 \mu \mathrm{m}$, Inset $50 \mu \mathrm{m}$. The wound edge is indicated with a black broken line. $\mathbf{b} \mathrm{H} \& \mathrm{E}$ and immunostaining of MCL-1 in skins 3 days post wounding in DMSO or inhibitor treated mice as indicated. Representative image of $n=15$ mice per group, Scale bar: $200 \mu \mathrm{m}$. The wound edge is indicated with a black broken line. c Quantification of MCL-1 positive cells in the area surrounding the wound site. $n=15$ mice per group, each dot represents an individual mouse, mean values \pm s.e.m.; ${ }^{*} p<0.0001$ compared to DMSO; Tukey corrected one-way ANOVA. d Quantification of dorsal wound coverage in DMSO or inhibitor treated mice over a 7-day period. $N \geq 2$ mice per group, each dot represents an individual mouse, mean values \pm s.e.m.; Tukey corrected one-way ANOVA at day 7.

surrounding cells. This mechanism fits the concept of persistence, which has emerged to explain transient, non-heritable resistance of tumour cells to therapy ${ }^{38}$. Cancer cells that transiently evade cell death, called persister cells, blunt the effectiveness of chemotherapy and provide a cell pool from which drug-resistant tumours may arise. The mechanism we describe herein represents a non-cell autonomous way of generating persistence (via FGF2 release) that requires activation of the core apoptotic pathway. Suggestive of its relevance in vivo, we find that in certain cancer types there is a correlation between FGF-signalling, anti-apoptotic BCL-2 protein expression and poor prognosis. Importantly, we find that inhibition of FGF receptor signalling or of downstream MEK-ERK signalling greatly impedes the ability of stressed cells to promote survival in a non-cell autonomous manner. Our data show that apoptotic resistance is transient lasting multiple days, after which cells become sensitised again to BH3-mimetics. Extrapolating these findings to a clinical setting, one possibility may be to combine apoptosis inducing cancer therapy with FGFR inhibitors. Additionally, our data suggest that intermittent dosing of apoptosis inducing therapies, employing so-called drug holidays, may help circumvent apoptotic resistance.

Some molecular and cellular mechanisms are known to be shared by both wounds and cancers, bringing forward the concept of tumours as over-healing ${ }^{39}$. Tissue integrity is essential for the proper functioning of any multicellular organism. To maintain this integrity, breaches have to be refilled, for instance by increased migration and proliferation. Epithelial tissue like skin is often subjected to injury, and efficient repair is necessary to close the wound and to restore proper barrier functions. This wound repair is facilitated by growth factors like FGF2 to promote cell proliferation and angiogenesis ${ }^{40,41}$. Interestingly, secretion of FGF2 was shown to be induced by shear stress ${ }^{42}$, and activation of ERK signalling is observed in several instances of tissue regeneration ${ }^{43,44}$. Pro-survival ERK signalling engaged by EGF released by apoptotic cells has recently been found to promote tissue integrity 45,46 . Importantly, while we find that FGF2 can be released from viable cells under conditions of apoptotic stress, EGF release required apoptosis. This highlights that proapoptotic signalling can engage multiple pro-survival mechanisms. The role of BCL-2 proteins in skin injury has not been extensively studied, however MCL-1 seems to be involved in keratinocyte survival and differentiation ${ }^{47}$. Our data now provide a potential connection between FGF2 and MCL-1 in wound healing: In response to cell stress due to injury, cells secrete FGF2 to induce pro-survival MCL-1 expression in neighbouring cells and to increase their apoptotic threshold. Increasing resistance to death in this setting might have several reasons, for example to limit the sustained damage by preventing extensive cell death or to promote regeneration of the tissue by protecting heavily proliferating cells from death. Inhibiting FGF signalling during wounding or decreasing the apoptotic threshold with $\mathrm{BH} 3-$ mimetics therefore delays wound healing. An intriguing avenue 
for the future would be to examine how signals released from apoptotic cells can be harnessed to facilitate tissue regeneration.

A remaining question is how apoptotic stress leads to FGF2 release in dying cells. FGF2 is secreted from cells in a nonconventional manner that remains to be fully elucidated ${ }^{48}$. Importantly, while FGF2 release occurs during caspasedependent apoptosis, it is not dependent on apoptosis - neither inhibition of caspase function nor MOMP prevents FGF2 release. This suggests that neutralisation of anti-apoptotic BCL-2 proteins exerts a non-lethal signalling function, leading to FGF2 release. Indeed, a variety of non-apoptotic functions for BCL-2 have been reported previously, for instance roles in calcium signalling or metabolism ${ }^{49}$. Alternatively, BH3-only proteins may be responsible for this FGF2 release. FGF2 is secreted in a non-canonical manner, which involves its binding to $\mathrm{PI}(4,5) \mathrm{P}_{2}$ at the plasma membrane followed by insertion of FGF2 oligomers through the membrane ${ }^{50}$. Although tBID was described to be able to interact with various membranes like artificial liposomes ${ }^{51}$, lysosomes ${ }^{52}$ or mitochondria ${ }^{53}$, it remains to be determined if this function of tBID can be extended to FGF2 secretion.

Our data further emphasise that cell death exerts a plethora of non-cell autonomous effects. These include context dependent pro-proliferative, inflammatory and apoptotic activities ${ }^{54-56}$. The mechanism we describe here represents an FGF-driven pro-survival effect of apoptotic stress. As discussed, this effect may have important implications for dictating therapeutic efficacy of apoptosis-inducing cancer therapy. Beyond this, we show that apoptotic stress induced survival signalling may also have a physiological role linking tissue stress to tissue repair.

\section{Methods}

Cell lines and chemicals. HeLa and 293T cells were purchased from ATCC, HeLa tBID2A BCL-2 were previously described ${ }^{9}$, MRC5 and IMR90 cells were a gift from Peter Adams, Beatson Institute, and CWR-R1 cells were a gift from Arnaud Blomme, Beatson Institute. Cell lines were not authenticated. Cells were regularly tested negative for mycoplasm. HeLa, HeLa tBID2A BCL-2 cells ${ }^{9}$, IMR90, MRC-5 and 293 T cells were cultured in DMEM high-glucose medium supplemented with $10 \%$ FCS and $2 \mathrm{mM}$ glutamine. CWR-R1 cells were cultured in RPMI high-glucose medium supplemented with $10 \%$ FCS and 2 mM glutamine. MRC5 and IMR90 cells were cultured in $3 \%$ oxygen. To select for venetoclax resistant cells, HeLa tBID2A BCL-2 cells were cultured continuously in the indicated dose of venetoclax for 14 days or cultured for $8 \mathrm{~h}$ in venetoclax followed by $16 \mathrm{~h}$ normal medium daily. The following drugs and chemicals were used: ABT-199/venetoclax (AdooQ BioScience, A12500-50), ABT-263/Navitoclax (ApexBio, A3007), ABT-737 (ApexBio, A8193), Actinomycin D (Calbiochem, 114666), AZD4547 (Selleck, S2801), Chir99021 (GSK3 inhibitor, final concentration $3 \mu \mathrm{M}$, Gift from D. Murphy), Cycloheximide (Sigma, 1810), Doxorubiucin (Sigma D1515), EGF (Sigma, E4127), Etoposide (Sigma, E1383), FGF2 (Thermo, PHG0263), GSK690693 (AKT inhibitor, final concentration $1 \mathrm{uM}$, gift from Daniel Murphy, University of Glasgow), Paclitaxel (Sigma, T7191), PRN1371 (Selleck, S8578), Propidium iodide (Sigma, P4170), Proteinase K (Thermo, 25530049), Pyr41 (E1 Ubiquitin ligase inhibitor, final concentration $50 \mu \mathrm{M}$, Sigma, N2915), qVD-OPh (AdooQ BioScience, A14915-25), Rapamycin (mTOR inhibitor, final concentration $1 \mu \mathrm{M}$, Santa Cruz, sc-3504), S55746 (ProbeChem, PC-63502), S63845 (Chemgood, C1370), Sytox Green (Thermo, S7020), Syto 21 (Sigma, S7556) and trametinib (MEK inhibitor, Cambridge Bioscience, HY-10999).

Lentiviral transduction. CRISPR-Cas9-based genome editing was performed using LentiCRISPRv2-puro (Addgene \#52961) or LentiCRISPRv2-blasti ${ }^{9}$ using the following guide sequences: hBAX, 5'-AGTAGAAAAGGGCGACAACC-3'; hBAK, 5'-GCCATGCTGGTAGACGTGTA-3'; hERK1, 5'-CAGAATATGTGGCCACA CGT-3'; hERK2, 5'-AGTAGGTCTGATGTTCGAAG-3'; hFGF2.1, 5' -TATGCA AGTCCAACGCACTG $-3^{\prime}$ and hFGF2.2, $5^{\prime}$-CGAGCTACTTAGACGCACCC- $3^{\prime}$.

For stable cell line generation, $5^{*} 10^{\wedge} 6293 \mathrm{FT}$ cells were transfected in $10 \mathrm{~cm}$ dishes using $4 \mu \mathrm{g}$ polyethylenimine (PEI, Polysciences) per $\mu \mathrm{g}$ plasmid DNA with lentiCRISPR_V2 (Addgene 52961): gag/pol (Addgene 14887): pUVSVG (Addgene $8454)$ at a 4:2:1 ratio. After 48 and $72 \mathrm{~h}$ of transfection, virus containing supernatant was filtered, supplemented with $1 \mu \mathrm{g} / \mathrm{ml}$ polybrene (Sigma) and added to 50.000 recipient cells in a 6 well plate. Selection with appropriate antibiotics $(1 \mu \mathrm{g} / \mathrm{ml}$ puromycin (Sigma) or $10 \mu \mathrm{g} / \mathrm{ml}$ blasticidin (InvivoGen) ) was started $24 \mathrm{~h}$ after the last infection and continued for one week ${ }^{57}$.
Supernatant assays. Generally, cells were treated for $3 \mathrm{~h}$ with $500 \mathrm{nM}$ venetoclax then the medium was replaced with regular growth medium. After an additional $45 \mathrm{~h}$ the supernatant was harvested, filtered and added onto recipient cells. For Proteinase $\mathrm{K}$ treatment, supernatant was treated with $200 \mu \mathrm{g} / \mathrm{ml}$ Proteinase $\mathrm{K}$ for $60 \mathrm{~min}$ at $50{ }^{\circ} \mathrm{C}$, followed by $5 \mathrm{~min}$ at $95^{\circ} \mathrm{C}$. After cooling down, the treated supernatant was added to recipient cells. For centrifugal filtration, supernatant was filtered and added into an Amicon Ultra $15 \mathrm{ml}$ tube (Merck) with a $3 \mathrm{kDa}$ cut-off. After spinning at $4000 \mathrm{~g}$ for $60 \mathrm{~min}$, the concentrate was diluted with regular growth medium to its original volume and the concentrate or the flowthrough was added onto recipient cells. The FGF2 ELISA was performed using the Human FGFbasic ELISA MAX Deluxe (Biolegend) according to the manufacturer's instructions after 50x concentration of the supernatant by centrifugal filtration (see above). The final FGF2 concentration was determined using a standard of recombinant FGF2, taking into account the concentration step.

Plasmid and siRNA transfection. For plasmid transfection, Lipofectamine 2000 was used according to the manufacturer's instructions. Transfection of siRNA was performed using Oligofectamine according to the manufacturer's instructions. The following siGENOME SMARTpool siRNAs from Dharmacon were used: Nontargeting, D0012061305; hFGFR1, M-003131-03-0005; hFGFR3, M-003133-010005 and hFGFR4, M-003134-02-0005.

Western blotting. Cell lysates were prepared using lysis buffer (1\% NP-40, $0.1 \%$ SDS, $1 \mathrm{mM}$ EDTA, $150 \mathrm{mM} \mathrm{NaCl}, 50 \mathrm{mM}$ Tris pH7.5, supplemented with Complete Protease Inhibitors (Roche) and PhosSTOP (Roche)). Protein concentration was determined with Bio-Rad Protein Assay Dye (5000006), and lysates were separated by SDS-PAGE followed by blotting onto nitrocellulose membranes and incubation with primary antibody (1:1000 in 5\% milk) overnight. After washing in TBS/T and incubation with secondary antibody (Li-Cor IRDye 800CW donkey anti-rabbit, 926-32213, dilution 1:20000), blots were developed on a $\mathrm{Li}$-Cor Odyssey CLx system and acquired using Imagestudio (Li-Cor). The following primary antibodies were used: Actin (A4700, Sigma), BAK (12105, Cell Signaling), BAX (2772, Cell Signaling), BCL-2 (4223, Cell Signaling), ERK1/2 (4695, Cell Signaling), basic FGF (20102, Cell Signaling), FGFR1 (9740, Cell Signaling), FGFR3 (4574, Cell Signaling), FGFR4 (8562, Cell Signaling), GFP (In house), HSP60 (4870, Cell Signaling), MCL-1 (5453, Cell Signaling), pERK1/2 (4370, Cell Signaling), Caspase 3 (9662, Cell Signaling), Caspase 9 (9502, Cell Signaling), PARP1 (9532, Cell Signaling) alpha-Tubulin (T5168, Sigma) and cleaved Caspase 3 (9664, Cell Signaling).

Quantitative RT-PCR. RNA from cultured cells was isolated with the GeneJET RNA purification kit according to the manufacturer's instructions. cDNA synthesis was performed according to the manufacturer's instructions using the High Capacity cDNA Reverse Transcription Kit (Thermo), and qPCR was performed with the Brilliant III Ultra-Fast SYBR Green qPCR Master Mix (Agilent Technologies) on a QuantStudio 3 machine (Applied Biosystems) with the following programme: $3 \mathrm{~min}$ at $95^{\circ} \mathrm{C}, 40 \mathrm{cycles}$ of $20 \mathrm{~s}$ at $95^{\circ} \mathrm{C}, 30 \mathrm{~s}$ at $57^{\circ} \mathrm{C}, 30 \mathrm{~s}$ at $72^{\circ} \mathrm{C}$ and a final $5 \mathrm{~min}$ at $72^{\circ} \mathrm{C}$. Results were analysed using the $2^{-\Delta \Delta \mathrm{Ct}}$ method. Primer sequences can be found in Supplementary Table 1.

Cell death assays. Short-term cell death was determined with an Incucyte FLR or Zoom imaging system (Essen Bioscience) ${ }^{58}$. Cells were treated as indicated in the Figure legend together with $30 \mathrm{nM}$ Sytox green and imaged every 1 or $2 \mathrm{~h}$. Analysis was performed using the Incucyte software and the number of dead (Sytox green positive) cells was normalised to the confluency at $t=0$. Alternatively, cells were pre-treated for $1 \mathrm{~h}$ with $50 \mathrm{nM}$ Syto 21 , followed by cytotoxic treatments as indicated together with $5 \mu \mathrm{g} / \mathrm{ml}$ propidium iodide. Long-term colony formation assay was performed by plating 1000 cells per 6 well and treatment as described in the Figure legend. After $48 \mathrm{~h}$ of treatment with supernatant, the medium was changed to $2.5 \mu \mathrm{M}$ venetoclax/S63845. Medium was changed to regular growth medium after an additional $48 \mathrm{~h}$, and resulting colonies were stained with crystal violet after an additional 5 days. Cell death analysis via FACS was performed using Annexin V - propidium iodide staining ${ }^{59}$. In short, treated cells were harvested and stained with $5 \mu \mathrm{g} / \mathrm{ml}$ propidium iodide and Annexin V (Biolegend) in Annexin V-binding buffer (10 mM Hepes pH 7.4, $140 \mathrm{mM} \mathrm{NaCl}, 2.5 \mathrm{mM} \mathrm{CaCl}_{2}$ ) for $15 \mathrm{~min}$. Flow cytometry was conducted on a BD FACSCalibur machine with BD CellQuest software and analysed using Flowing software; cells negative for propidium iodide and Annexin V were considered alive.

Membrane ligand array. Supernatant was harvested from HeLa tBID2A cells as described above and used to probe a Human Growth Factor Antibody Array (Abcam, ab134002) according to the manufacturer's instructions.

Bioinformatic analysis. Mutation, survival and gene expression data (Thymoma TCGA Firehose Legacy) was downloaded from cBioPortal (www.cbioportal.org). Samples with alterations (Deletions, mutations or amplifications) in components of the FGFR signalling pathway (FGFR1, FGFR3, GRB2, FRS2, SOS1, HRAS, RAF1, MAPK1, MAPK3) were removed. The FGF score was determined by averaging the 
expression of the FGF2 induced gene set (CDH2, CDX4, FES, FGF2, FRS2, FYN, HOXA10, INHBA, MAP2K1, MAPK8, MMP7, PF4, RUNX2, SERPINF1) from the Harmonizome database (https://maayanlab.cloud/Harmonizome/gene_set/ fgf2induced/GeneRIF+Biological+Term+Annotations). Pearson correlation was calculated between FGF score and BCL-2 or MCL-1 expression. Samples were stratified into thirds based on FGF score, BCL-2 or MCL-1 expression. Survival was analysed comparing samples with high FGF score and high expression of BCL-2 or MCL-1 with low FGF score and low expression of BCL-2 and MCL-1. The used code is available as a supplementary software file (Supplementary Software 1).

Wound healing experiments. For all wound repair experiments, C57BL/6 J mice were sex and age matched (8-weeks-old) and randomly assigned to different treatment groups. Mice were first shaved and full-thickness $1 \mathrm{~cm}^{2}$ excision wounds were performed on the dorsal skin. Following wound infliction, mice were euthanized with $\mathrm{CO}_{2}$ at various time points and wounded skins were embedded in OCT for analysis. Housing, care and wounding experiments were approved by the ethical committee of the Technion - Israel Institute of Technology

Immunofluorescence and Hematoxylin and Eosin staining. Skins frozen in OCT were sectioned at $12 \mu \mathrm{m}$ and fixed in $4 \%$ paraformaldehyde for $20 \mathrm{~min}$ at room temperature. Samples were then blocked for $2 \mathrm{~h}$, followed by incubation with primary antibodies diluted in blocking buffer overnight at $4{ }^{\circ} \mathrm{C}$. Following washing, samples were incubated with secondary antibodies (Alexa Fluor 488/546/633, Thermo) for $1 \mathrm{~h}$ at room temperature. The following primary antibodies were used: MCL-1 (1:800, 5453, Cell Signaling), Ki67 (1:100, 9882, eBioscience), Mcm2 (1:500, 4461, Abcam), active ItgB1 (1:100, BD, 550531) and Alexa Fluor 568 phalloidin. Sample analysis was performed on a Zeiss LSM 880 confocal microscope and analysed using Zen software. Sections were first treated with Hematoxylin followed by $\mathrm{H}_{2} \mathrm{O}$, differentiator $(0.3 \%$ alcoholic $\mathrm{HCl}), \mathrm{H}_{2} \mathrm{O}, 95 \% \mathrm{EtOH}$, Eosin, $95 \% \mathrm{EtOH}$, twice in $100 \% \mathrm{EtOH}$ and three times in xylene before mounting in xylene based mounting medium.

Reporting summary. Further information on research design is available in the Nature Research Reporting Summary linked to this article.

\section{Data availability}

Source data are provided with this paper. The TCGA data used in this study are available in the cBioPortal database under [www.cbioportal.org/study/summary?id=thym_tcga], [https://www.cbioportal.org/study/summary?id=kich_tcga] and [https://www.cbioportal. org/study/summary?id=ucec_tcga]. The FGF score was based on the fgf2induced dataset from the Harmonizome [https://maayanlab.cloud/Harmonizome/gene set/fgf2induced/ GeneRIF+Biological+Term+Annotations]. The remaining data are available within the Article and Supplementary Information. Source data are provided with this paper.

\section{Code availability}

Bioinformatic analysis was conducted in $\mathrm{R}$, the used code is available as supplementary software file.

Received: 10 August 2020; Accepted: 8 October 2021;

Published online: 12 November 2021

\section{References}

1. Singh, R., Letai, A. \& Sarosiek, K. Regulation of apoptosis in health and disease: the balancing act of BCL-2 family proteins. Nat. Rev. Mol. Cell. Biol. https://doi.org/10.1038/s41580-018-0089-8 (2019).

2. Certo, M. et al. Mitochondria primed by death signals determine cellular addiction to antiapoptotic BCL-2 family members. Cancer Cell 9, 351-365 (2006).

3. Bock, F. J. \& Tait, S. W. G. Mitochondria as multifaceted regulators of cell death. Nat. Rev. Mol. Cell Biol. 21, 85-100 (2020).

4. Oltersdorf, T. et al. An inhibitor of $\mathrm{Bcl}-2$ family proteins induces regression of solid tumours. Nature 435, 677-681 (2005).

5. Souers, A. J. et al. ABT-199, a potent and selective BCL-2 inhibitor, achieves antitumor activity while sparing platelets. Nat. Med. 19, 202-208 (2013).

6. Roberts, A. W. et al. Targeting BCL2 with venetoclax in relapsed chronic lymphocytic leukemia. N. Engl. J. Med. 374, 311-322 (2016).

7. Wei, A. H. et al. Venetoclax combined with low-dose cytarabine for previously untreated patients with acute myeloid leukemia: results from a phase Ib/II study. J. Clin. Oncol. 37, 1277-1284 (2019).

8. DiNardo, C. D. et al. Safety and preliminary efficacy of venetoclax with decitabine or azacitidine in elderly patients with previously untreated acute myeloid leukaemia: a non-randomised, open-label, phase $1 \mathrm{~b}$ study. Lancet Oncol. 19, 216-228 (2018).

9. Lopez, J. et al. Mito-priming as a method to engineer Bcl-2 addiction. Nat. Commun. 7, https://doi.org/10.1038/ncomms10538 (2016).

10. Campbell, K. J. \& Tait, S. W. G. Targeting BCL-2 regulated apoptosis in cancer. Open Biol 8, https://doi.org/10.1098/rsob.180002 (2018).

11. Kotschy, A. et al. The MCL1 inhibitor S63845 is tolerable and effective in diverse cancer models. Nature 538, 477-482 (2016).

12. Wei, M. C. et al. Proapoptotic BAX and BAK: a requisite gateway to mitochondrial dysfunction and death. Science 292, 727-730 (2001).

13. Casara, P. et al. S55746 is a novel orally active BCL-2 selective and potent inhibitor that impairs hematological tumor growth. Oncotarget 9 , 20075-20088 (2018).

14. Townsend, K. J., Trusty, J. L., Traupman, M. A., Eastman, A. \& Craig, R. W. Expression of the antiapoptotic MCL1 gene product is regulated by a mitogen activated protein kinase-mediated pathway triggered through microtubule disruption and protein kinase C. Oncogene 17, 1223-1234 (1998).

15. Maurer, U., Charvet, C., Wagman, A. S., Dejardin, E. \& Green, D. R. Glycogen Synthase kinase-3 Regulates Mitochondrial Outer Membrane Permeabilization and Apoptosis by Destabilization of MCL-1. Mol. Cell 21, 749-760 (2006).

16. Mills, J. R. et al. mTORC1 promotes survival through translational control of Mcl-1. Proc. Natl Acad. Sci. USA 105, 10853-10858 (2008).

17. Pugazhenthi, S. et al. Akt/protein kinase B up-regulates Bcl-2 expression through cAMP-response element-binding protein. J. Biol. Chem. 275, 10761-10766 (2000).

18. Gilmartin, A. G. et al. GSK1120212 (JTP-74057) is an inhibitor of MEK activity and activation with favorable pharmacokinetic properties for sustained in vivo pathway inhibition. Clin. Cancer Res. 17, 989-1000 (2011).

19. Crews, C. M. \& Erikson, R. L. Purification of a murine protein-tyrosine/ threonine kinase that phosphorylates and activates the Erk-1 gene product: relationship to the fission yeast byrl gene product. Proc. Natl Acad. Sci. USA 89, 8205-8209 (1992).

20. Bernardo, A. S. et al. BRACHYURY and CDX2 mediate BMP-induced differentiation of human and mouse pluripotent stem cells into embryonic and extraembryonic lineages. Cell Stem Cell 9, 144-155 (2011).

21. Li, C., Scott, D. A., Hatch, E., Tian, X. \& Mansour, S. L. Dusp6 (Mkp3) is a negative feedback regulator of FGF-stimulated ERK signaling during mouse. Dev. Dev. 134, 167-176 (2007).

22. Lai, W. T., Krishnappa, V. \& Phinney, D. G. Fibroblast growth factor 2 (Fgf2) inhibits differentiation of mesenchymal stem cells by inducing Twist 2 and Spry4, blocking extracellular regulated kinase activation, and altering Fgf receptor expression levels. Stem Cells 29, 1102-1111 (2011).

23. Gavine, P. R. et al. AZD4547: an orally bioavailable, potent, and selective inhibitor of the fibroblast growth factor receptor tyrosine kinase family. Cancer Res. 72, 2045-2056 (2012)

24. Venetsanakos, E. et al. The irreversible covalent fibroblast growth factor receptor inhibitor PRN1371 exhibits sustained inhibition of FGFR after drug clearance. Mol. Cancer Ther. 16, 2668-2676 (2017).

25. Ornitz, D. M. \& Itoh, N. The fibroblast growth factor signaling pathway. Wiley Interdiscip. Rev. Dev. Biol. 4, 215-266 (2015).

26. Fuchs, Y. et al. Sept4/ARTS regulates stem cell apoptosis and skin regeneration. Science 341, 286-289 (2013).

27. Li, F. et al. Apoptotic cells activate the "Phoenix Rising" pathway to promote wound healing and tissue regeneration. Sci. Signal. 3, ra13-ra13 (2010).

28. Steiling, H. \& Werner, S. Fibroblast growth factors: key players in epithelial morphogenesis, repair and cytoprotection. Curr. Opin. Biotechnol. 14, 533-537 (2003)

29. Park, S. et al. Tissue-scale coordination of cellular behaviour promotes epidermal wound repair in live mice. Nat. Cell Biol. 19, 155-163 (2017)

30. Aragona, M. et al. Defining stem cell dynamics and migration during wound healing in mouse skin epidermis. Nat. Commun. 8, 14684 (2017).

31. Fernald, K. \& Kurokawa, M. Evading apoptosis in cancer. Trends Cell Biol. 23 620-633 (2013)

32. Tomlinson, D. C., Lamont, F. R., Shnyder, S. D. \& Knowles, M. A. Fibroblast growth factor receptor 1 promotes proliferation and survival via activation of the mitogen-activated protein kinase pathway in bladder cancer. Cancer Res. 69, 4613-4620 (2009).

33. Konig, A. et al. Basic fibroblast growth factor (bFGF) upregulates the expression of bcl-2 in B cell chronic lymphocytic leukemia cell lines resulting in delaying apoptosis. Leukemia 11, 258-265 (1997).

34. Blombery, P. et al. Acquisition of the recurrent Gly101Val mutation in BCL2 Confers resistance to venetoclax in patients with progressive chronic lymphocytic leukemia. Cancer Discov. 9, 342-353 (2019).

35. Blombery, P. et al. Multiple BCL2 mutations cooccurring with Gly101Val emerge in chronic lymphocytic leukemia progression on venetoclax. Blood 135, 773-777 (2020). 
36. Birkinshaw, R. W. et al. Structures of BCL-2 in complex with venetoclax reveal the molecular basis of resistance mutations. Nat. Commun. 10, 2385 (2019).

37. Blombery, P. et al. Characterization of a novel venetoclax resistance mutation (BCL2 Phe104Ile) observed in follicular lymphoma. Br. J. Haematol. 186, e188-e191 (2019).

38. Boumahdi, S. \& de Sauvage, F. J. The great escape: tumour cell plasticity in resistance to targeted therapy. Nat. Rev. Drug Discov. 19, 39-56 (2020).

39. Soteriou, D. \& Fuchs, Y. A matter of life and death: stem cell survival in tissue regeneration and tumour formation. Nat. Rev. Cancer 18, 187-201 (2018).

40. Meyer, M. et al. FGF receptors 1 and 2 are key regulators of keratinocyte migration in vitro and in wounded skin. J. Cell Sci. 125, 5690-5701 (2012).

41. Barrientos, S., Stojadinovic, O., Golinko, M. S., Brem, H. \& Tomic-Canic, M. Growth factors and cytokines in wound healing. Wound Repair Regen. 16, 585-601 (2008).

42. Gloe, T., Sohn, H. Y., Meininger, G. A. \& Pohl, U. Shear stress-induced release of basic fibroblast growth factor from endothelial cells is mediated by matrix interaction via integrin alpha(v)beta3. J. Biol. Chem. 277, 23453-23458 (2002).

43. De Simone, A. et al. Control of osteoblast regeneration by a train of Erk activity waves. Nature 590, 129-133 (2021).

44. Kinoshita, N. et al. Mechanical stress regulates epithelial tissue integrity and stiffness through the FGFR/Erk2 signaling pathway during embryogenesis. Cell Rep. 30, 3875-3888.e3873 (2020).

45. Gagliardi, P. A. et al. Collective ERK/Akt activity waves orchestrate epithelial homeostasis by driving apoptosis-induced survival. Dev. Cell, https://doi.org/ 10.1016/j.devcel.2021.05.007 (2021)

46. Valon, L. et al. Robustness of epithelial sealing is an emerging property of local ERK feedback driven by cell elimination. Dev. Cell, https://doi.org/10.1016/ j.devcel.2021.05.006 (2021).

47. Sitailo, L. A., Jerome-Morais, A. \& Denning, M. F. Mcl-1 functions as major epidermal survival protein required for proper keratinocyte differentiation. $J$. Invest. Dermatol. 129, 1351-1360 (2009).

48. Brough, D., Pelegrin, P. \& Nickel, W. An emerging case for membrane pore formation as a common mechanism for the unconventional secretion of FGF2 and IL-1beta. J. Cell Sci. 130, 3197-3202 (2017).

49. Vanden Abeele, F. et al. Bcl-2-dependent modulation of $\mathrm{Ca}(2+)$ homeostasis and store-operated channels in prostate cancer cells. Cancer Cell 1, 169-179 (2002).

50. Dimou, E. et al. Single event visualization of unconventional secretion of FGF2. J. Cell Biol. 218, 683-699 (2019).

51. Bleicken, S., Garcia-Saez, A. J., Conte, E. \& Bordignon, E. Dynamic interaction of cBid with detergents, liposomes and mitochondria. PLoS ONE 7, e35910 (2012).

52. Zhao, K. et al. Phosphatidic acid mediates the targeting of tBid to induce lysosomal membrane permeabilization and apoptosis. J. Lipid Res. 53, 2102-2114 (2012).

53. Grinberg, M. et al. tBID Homooligomerizes in the mitochondrial membrane to induce apoptosis. J. Biol. Chem. 277, 12237-12245 (2002).

54. Perez-Garijo, A. \& Steller, H. Spreading the word: non-autonomous effects of apoptosis during development, regeneration and disease. Development 142, 3253-3262 (2015).

55. Lohard, S. et al. STING-dependent paracriny shapes apoptotic priming of breast tumors in response to anti-mitotic treatment. Nat. Commun. 11, 259 (2020).

56. Koren, E. \& Fuchs, Y. Modes of regulated cell death in cancer. Cancer Disco. 11, 245-265 (2021)

57. Giampazolias, E. et al. Mitochondrial permeabilization engages NF-kBdependent anti-tumour activity under caspase deficiency. Nat. Cell Biol. 19, 1116-1129 (2017).
58. Riley, J. S. et al. Mitochondrial inner membrane permeabilisation enables mtDNA release during apoptosis. EMBO J. 37, https://doi.org/10.15252/ embj.201899238 (2018).

59. Bock, F. J. et al. Loss of PIDD limits NF-kappaB activation and cytokine production but not cell survival or transformation after DNA damage. Cell Death Differ. 20, 546-557 (2013).

\section{Acknowledgements}

This work was supported by Cancer Research UK grant C40872/A2014 (S.W.G.T), CRUK core funding A29799 to K.B. and a Tenovus small pilot grant (F.J.B). We thank Joel Riley and Catherine Winchester for reviewing the manuscript and all members of the Tait laboratory for helpful suggestions as well as Hasan Uludağ (University of Alberta, Canada) for reagents. We would like to thank the Core Services and Advanced Technologies at the Cancer Research UK Beatson Institute (C596/A17196), with particular thanks to the Beatson Advanced Imaging Resource, Biological Services Unit, Histology and Molecular Technologies. Schematic figures were created using BioRender.com.

\section{Author contributions}

Conceived the study and designed the work plan: F.J.B., K.B. and S.W.G.T.; Experimental work: F.J.B., A.L.K., D.A., J.A., K.J.C., C.C., D.Z., E.S. and E.K.; Data analysis: F.J.B., E.S. Y.F. and S.W.G.T.; Manuscript writing: F.J.B., Y.F. and S.W.G.T.

\section{Competing interests}

The authors declare no competing interests.

\section{Additional information}

Supplementary information The online version contains supplementary material available at https://doi.org/10.1038/s41467-021-26613-0.

Correspondence and requests for materials should be addressed to Florian J. Bock or Stephen W. G. Tait.

Peer review information Nature Communications thanks Richard Grose and Seamus Martin for their contribution to the peer review of this work. Peer reviewer reports are available.

Reprints and permission information is available at http://www.nature.com/reprints

Publisher's note Springer Nature remains neutral with regard to jurisdictional claims in published maps and institutional affiliations.

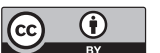

Open Access This article is licensed under a Creative Commons Attribution 4.0 International License, which permits use, sharing, adaptation, distribution and reproduction in any medium or format, as long as you give appropriate credit to the original author(s) and the source, provide a link to the Creative Commons license, and indicate if changes were made. The images or other third party material in this article are included in the article's Creative Commons license, unless indicated otherwise in a credit line to the material. If material is not included in the article's Creative Commons license and your intended use is not permitted by statutory regulation or exceeds the permitted use, you will need to obtain permission directly from the copyright holder. To view a copy of this license, visit http://creativecommons.org/ licenses/by/4.0\%

(C) The Author(s) 2021 